\title{
Hepatic neuregulin 4 signaling defines an endocrine checkpoint for steatosis-to-NASH progression
}

\author{
Liang Guo, ${ }^{1,2}$ Peng Zhang, ${ }^{1}$ Zhimin Chen, ${ }^{1}$ Houjun Xia, ${ }^{3}$ Siming Li, ${ }^{1}$ Yanqiao Zhang, ${ }^{4}$ Sune Kobberup, ${ }^{5}$ \\ Weiping Zou, ${ }^{3}$ and Jiandie D. Lin ${ }^{1}$
}

'Life Sciences Institute and Department of Cell \& Developmental Biology, University of Michigan Medical Center, Ann Arbor, Michigan, USA. Key Laboratory of Metabolism and Molecular Medicine,

Ministry of Education, and Department of Biochemistry and Molecular Biology, Fudan University Shanghai Medical College, Shanghai, China. ${ }^{3}$ Department of Surgery, University of Michigan School of

Medicine, Ann Arbor, Michigan, USA. ${ }^{4}$ Department of Integrative Medical Sciences, Northeast Ohio Medical University, Rootstown, Ohio, USA. ${ }^{5}$ Metabolic Disease Research, Novo Nordisk, Maaloev, Denmark.

\begin{abstract}
Nonalcoholic steatohepatitis (NASH) is characterized by progressive liver injury, inflammation, and fibrosis; however, the mechanisms that govern the transition from hepatic steatosis, which is relatively benign, to NASH remain poorly defined. Neuregulin 4 (Nrg4) is an adipose tissue-enriched endocrine factor that elicits beneficial metabolic effects in obesity. Here, we show that Nrg4 is a key component of an endocrine checkpoint that preserves hepatocyte health and counters diet-induced NASH in mice. Nrg4 deficiency accelerated liver injury, fibrosis, inflammation, and cell death in a mouse model of NASH. In contrast, transgenic expression of Nrg4 in adipose tissue alleviated diet-induced NASH. Nrg4 attenuated hepatocyte death in a cell-autonomous manner by blocking ubiquitination and proteasomal degradation of c-FLIP $\mathrm{L}_{\mathrm{L}}$ a negative regulator of cell death. Adeno-associated virus-mediated (AAV-mediated) rescue of hepatic c-FLIP $\mathrm{L}_{\mathrm{L}}$ expression in Nrg4-deficent mice functionally restored the brake for steatosis to NASH transition. Thus, hepatic Nrg4 signaling serves as an endocrine checkpoint for steatosis-to-NASH progression by activating a cytoprotective pathway to counter stress-induced liver injury.
\end{abstract}

\section{Introduction}

Nonalcoholic fatty liver disease (NAFLD) represents a spectrum of liver disorders linked to metabolic syndrome, ranging from simple fatty liver to more severe nonalcoholic steatohepatitis (NASH) (1-3). It has been estimated that over a third of the adult population and approximately $5 \%-10 \%$ of children in the US develop fatty liver. Hepatic steatosis results from a combination of adipose tissue dysfunction and dysregulation of hepatic lipid metabolism in an insulin-resistant state. NASH is characterized by the presence of persistent liver injury, chronic inflammation, and liver fibrosis. The latter is a wound-healing response that is activated following liver injury and potentially reversible at early stages (4). The transition from relatively benign hepatic steatosis to NASH marks a critical step in NAFLD progression that has important clinical implications, particularly the risk for end-stage liver disease, such as cirrhosis and hepatocellular carcinoma $(5,6)$. Multiple pathogenic drivers have been implicated in NASH initiation and progression, including oxidative stress, mitochondrial dysfunction, lipotoxicity, and immune cell activation (7-10). Despite this, the physiological mechanisms that defend against NASH progression remain poorly defined.

Liver injury as a result of hepatocyte death is at the center of many chronic liver diseases. Clinical and animal model studies have demonstrated that hepatocyte death is an important driver of liver disease progression (11-13). Aberrantly elevated liver

Conflict of interest: This work was partially supported by a research grant from Novo Nordisk.

Submitted: July 17, 2017; Accepted: September 26, 2017.

Reference information: J Clin Invest. 2017;127(12):4449-4461.

https://doi.org/10.1172/JCI96324. cell death is linked to activation of liver regeneration and wound repair program, resulting in persistent inflammation and liver fibrosis that increase risk for cirrhosis and liver cancer. Several forms of hepatocyte death have been observed in the liver, including apoptosis, necrosis, and necroptosis. Hepatocyte apoptosis is a well-recognized cell death pathway in NASH. Mice with genetic deletion of caspase 8 in hepatocytes exhibited attenuated liver injury, inflammation, and fibrosis following methionine/cholinedeficient (MCD) diet feeding (14). Caspase inhibitors have been shown to be protective in animal models of NASH $(15,16)$. Necroptosis is programmed necrosis that incorporates features of both apoptosis and necrosis $(17,18)$. The execution of necroptosis requires receptor-interacting protein 3 (RIPK3) and its substrate, mixed lineage kinase domain-like protein (MLKL). Upon RIPK3 activation, MLKL undergoes phosphorylation, oligomerization, and translocation to the plasma membrane, leading to membrane permeabilization and cell death. Necroptosis releases intracellular danger-associated molecular patterns, such as high-mobility group B1 (HMGB1), that have potential to trigger robust inflammatory response $(13,19)$. RIPK3 deficiency attenuates liver injury, steatosis, inflammation, and fibrosis in an MCD diet-induced NASH mouse model, implicating necroptosis in the liver as a contributor to NASH $(20,21)$. As such, targeting hepatocyte cell death may provide an effective approach to alleviating or reversing NASH progression (22).

Neuregulin $4(\mathrm{Nrg} 4)$ is a member of the EGF-like family of extracellular ligands that is abundantly expressed in adipose tissue (23-25). We previously demonstrated that Nrg4 binds to hepatocytes, activates ErbB3 and ErbB4 receptor tyrosine kinases, and attenuates hepatic lipogenic response (25). Nrg4 deficiency exacerbates, whereas its Tg expression protects mice from, diet-induced 
metabolic disorders. Interestingly, plasma Nrg4 levels inversely correlated with metabolic syndrome and NAFLD in 2 recent studies $(26,27)$. Recent genome-wide association studies indicate that polymorphisms in the ERBB4 locus are associated with obesity, diabetes, and diabetic nephropathy (28-30), illustrating a potential contribution of the Nrg4/ErbB4 pathway to the pathogenesis of metabolic disorders. Despite this, whether Nrg4 signaling regulates the transition from hepatic steatosis to NASH has not been explored. Using a diet-induced NASH model, we uncovered an unexpected function of $\mathrm{Nrg} 4$ in protecting hepatocytes from stress-induced cell death and attenuating NASH in mice. Mechanistically, Nrg4 exerts its cytoprotective effects on hepatocytes through attenuating ubiquitination and proteasomal degradation of cellular FADD-like apoptosis regulator (CFLAR, referred to as c-FLIP ${ }_{L}$ hereafter), a negative regulator of apoptotic and necroptotic cell death $(31,32)$. These observations establish hepatic Nrg4 signaling as an endocrine checkpoint for steatosis-to-NASH progression and reveal a potential target for therapeutic intervention.

\section{Results}

Human and mouse NASH is linked to increased apoptosis and necroptosis in the liver. Hepatocyte death is at the center of the transition from relatively benign hepatic steatosis to NASH. The latter is characterized by persistent liver injury, chronic inflammation, and progressive liver fibrosis. Despite the prominent role of cell death in NASH pathogenesis, the physiological cues that preserve hepatocyte health and tissue homeostasis of the liver under metabolic stress conditions remain poorly understood. To address this, we first investigated the nature of hepatocyte death in human NASH patients and in a mouse model of diet-induced NASH. Compared with non-NASH individuals, the levels of cleaved caspase 3 (CASP3) and phosphorylated MLKL (p-MLKL-S358), molecular markers of apoptosis and necroptosis, respectively, were markedly elevated in liver biopsies from a cohort of NASH patients (33) (Figure 1A). The induction of cell death in NASH livers was associated with increased phosphorylation of JNK1/2 and reduced protein levels of $\mathrm{c}-\mathrm{FLIP}_{\mathrm{L}}$, an inhibitor of apoptotic and necroptotic cell death. Quantitative PCR (qPCR) gene-expression analysis indicated that mRNA expression of genes involved in inflammation and fibrosis was markedly increased in the liver from NASH patients (Figure 1B).

We next evaluated a recently described high-fat high-fructose diet (NASH diet) in inducing key aspects of NASH pathology in mice (34). We fed a cohort of WT C57BL/6J mice standard chow or NASH diet for approximately 20 weeks. NASH diet-fed mice developed obesity and severe hepatic steatosis (Figure 1C and Supplemental Figure 1, A and B; supplemental material available online with this article; https://doi.org/10.1172/JCI96324DS1). Hepatic lipid droplet number and size were markedly increased following NASH diet feeding (Supplemental Figure 1, C and D). Compared with chow, plasma levels of alanine aminotransferase (ALT) and aspartate aminotransferase (AST) were markedly higher in mice fed a NASH diet (Figure 1D). Plasma concentration of high-mobility group box 1 (HMGB1), a danger-associated molecular pattern released by dead cells, was also significantly higher in the NASH group than control (Figure 1D). Sirius red staining of liver sections indicated that NASH diet-fed mice developed peri- cellular liver fibrosis that was associated with induction of genes involved in inflammation and fibrosis, increased abundance of F4/80-positive macrophages, and the presence of TUNEL-positive cells (Figure 1, C and E, and Supplemental Figure 1, E and F). Immunoblotting analysis of liver lysates revealed that CASP3 and poly(ADP-ribose) polymerase (PARP) cleavage (apoptosis) and MLKL phosphorylation (necroptosis) were induced by NASH diet feeding (Figure 1F). Similarly to what occurred in human NASH livers, phosphorylation of JNK was increased, whereas c-FLIP ${ }_{L}$ protein levels were reduced in the livers from NASH diet-fed mice. In contrast, protein levels of the short isoform c-FLIP $\mathrm{R}_{\mathrm{R}}$ remained similar. These observations demonstrate that NASH diet feeding recapitulates key pathologic events of human NASH in mice, including liver injury, inflammation, and fibrosis in the context of obesity. Interestingly, mRNA expression of $\mathrm{Nrg} 4$ in epididymal white adipose tissue (eWAT) and brown adipose tissue (BAT) was significantly decreased in NASH mice (Figure 1G). These results are consistent with recent work that demonstrates an inverse relationship between plasma Nrg4 levels and NASH scores in human patients (27). As Nrg4 is a fat-derived endocrine factor that acts on the liver, these findings raise the possibility that reduced Nrg4 signaling may contribute to the disruption of tissue homeostasis during steatosis-to-NASH progression.

$\mathrm{Nrg} 4$ deficiency exacerbates diet-induced $\mathrm{NASH}$ in mice. We previously demonstrated that $\mathrm{Nrg} 4$ protects mice from high-fat diet-induced (HFD-induced) metabolic disorders, including insulin resistance and hepatic steatosis (25). However, HFD feeding does not cause significant liver injury and fibrosis and is therefore inadequate for interrogating the role of $\mathrm{Nrg} 4$ in NASH. We next performed studies in Nrg4 gain- and loss-of-function mouse models in the context of diet-induced NASH. WT and Nrg4-KO mice were fed standard chow or NASH diet for approximately 20 weeks. Hepatic steatosis and fibrosis, plasma ALT and AST, and mRNA expression of genes involved in liver fibrosis and inflammation were similar between 2 groups under chow-fed conditions (Supplemental Figure 2, A-D). Following NASH diet feeding, Nrg4-KO mice had slightly higher liver triacylglycerol (TAG) content than control (Figure 2A). While the number of lipid droplets in hepatocytes was comparable, lipid droplets were slightly larger in mice lacking Nrg4 (Supplemental Figure 2, E and F). The exacerbation of hepatic steatosis by Nrg4 deficiency was less marked compared with what was observed in mice fed HFD, which does not contain fructose and cholesterol. Plasma levels of ALT, AST, and HMGB1, the biomarkers of liver injury, were significantly elevated in Nrg4KO mice following NASH feeding (Figure 2A). Nrg4-null mice exhibited more severe liver fibrosis, as revealed by sirius red staining and measurements of liver hydroxyproline content (Figure 2, $\mathrm{B}$ and C). Nrg4 deficiency also resulted in increased abundance of F4/80-positive macrophages and TUNEL-positive cells. Immunoblotting analysis indicated that CASP3 and PARP cleavage, MLKL phosphorylation, and JNK activation were strongly increased in Nrg4-KO mouse livers (Figure 2D). In contrast, c-FLIP ${ }_{\mathrm{L}}$ protein levels were markedly reduced by Nrg4 deficiency.

Transcriptional profiling analysis identified 2 clusters of upregulated and downregulated genes in Nrg4-null mouse livers. Gene ontology analysis indicated that the upregulated genes are highly enriched for extracellular matrix, wound healing, and colla- 
A

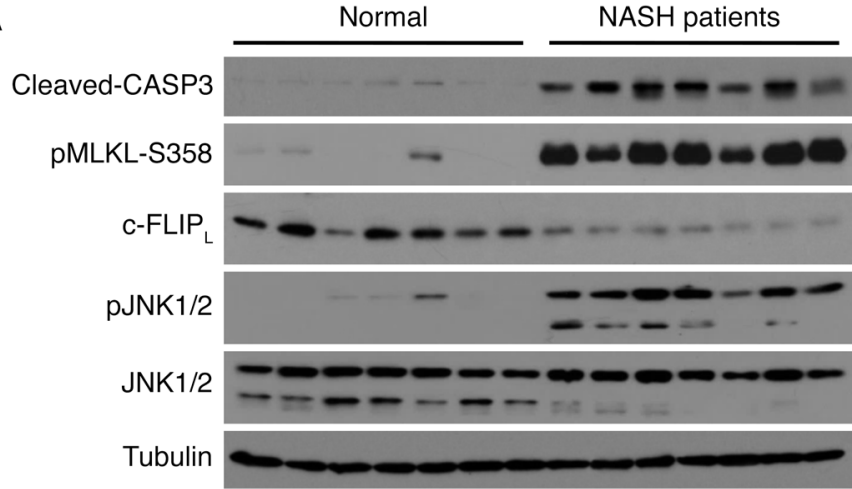

B

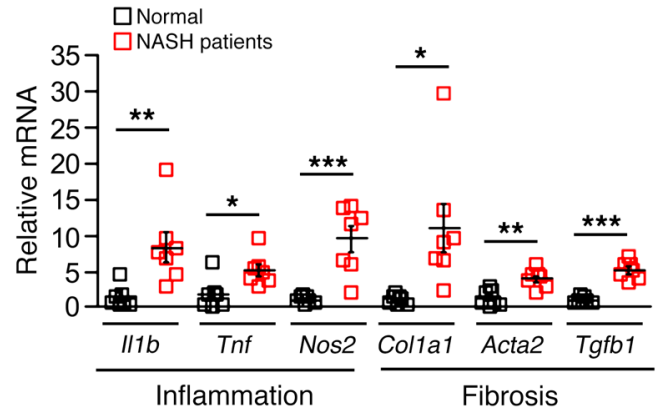

C<smiles>[135IH]</smiles>
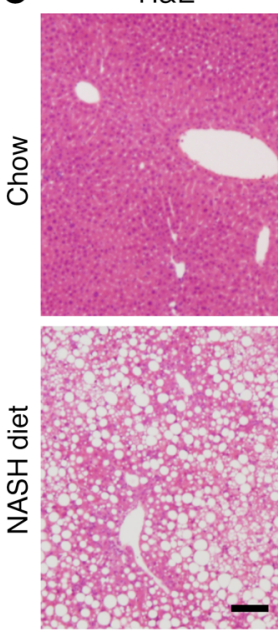

Sirius Red
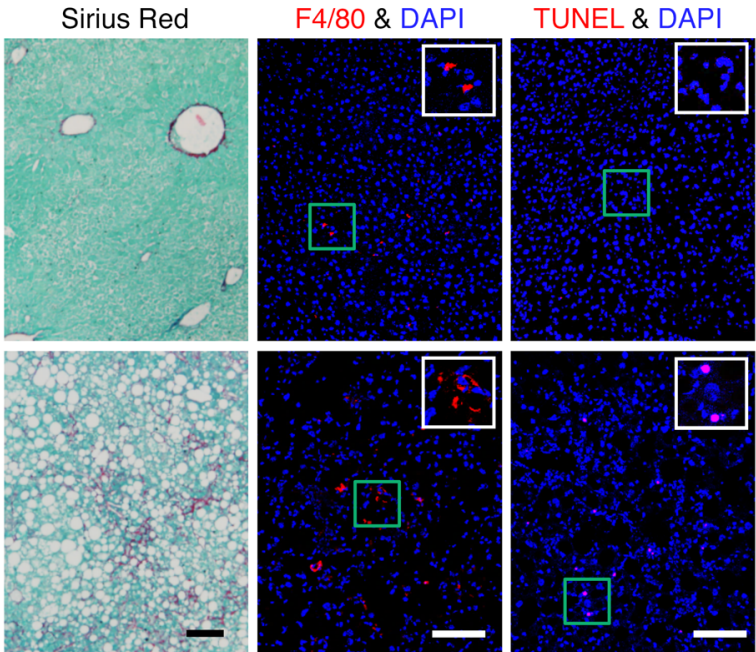

D
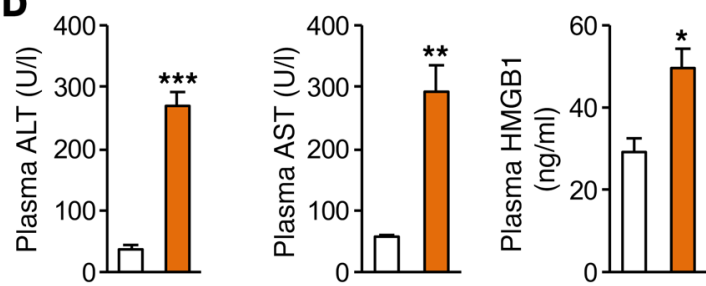

$\mathbf{F}$

Cleaved-Casp3

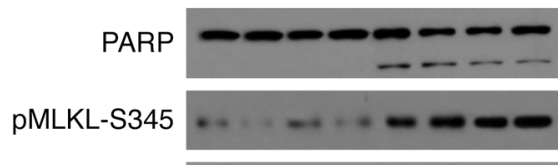

c-FLIP

C-FLIP

pJNK1/2

JNK1/2

Tubulin

G

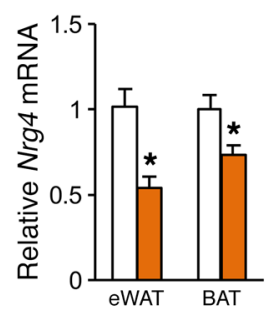

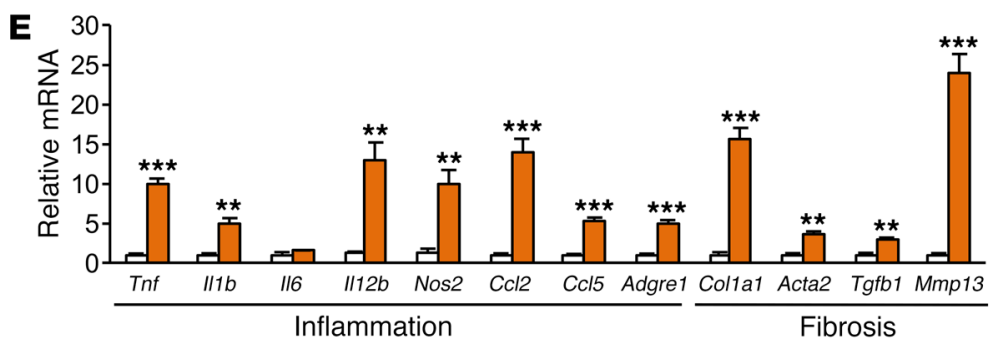

Figure 1. Human and mouse NASH are linked to induction of apoptosis and necroptosis in the liver. (A) Immunoblots of total liver lysates from normal individuals and NASH patients. (B) qPCR analysis of gene expression in normal $(n=7)$ and NASH $(n=7)$ human livers. Data represent mean \pm SEM. ${ }^{*} P<$ $0.05 ;{ }^{* *} P<0.01 ;{ }^{* *} P<0.001$, 2-tailed unpaired Student's $t$ test. (C) H\&E, sirius red, F4/80 immunofluorescence, and TUNEL staining of liver sections from male C57BL/6) mice fed chow or NASH diet. Scale bars: $100 \mu \mathrm{m}$. (D) Plasma ALT, AST, and HMCB1 levels in mice fed chow ( $n=4)$ or NASH diet ( $n=4)$. Data represent mean \pm SEM. ${ }^{*} P<0.05 ;{ }^{* *} P<0.01$; ${ }^{* *} P<0.001$, 2-tailed unpaired Student's $t$ test. (E) qPCR analysis of hepatic gene expression. Data represent mean \pm SEM. ${ }^{* *} P<0.01 ;{ }^{* *} P<0.001$, 2-tailed unpaired Student's $t$ test. (F) Immunoblots of total liver lysates from mice fed chow or NASH diet. (G) qPCR analysis of Nrg4 expression in eWAT and BAT. Data represent mean \pm SEM. ${ }^{*} P<0.05,2$-tailed unpaired Student's $t$ test.

gen, whereas the downregulated cluster was enriched for genes involved in lipid metabolism (Figure 2E). Consistently, mRNA expression of genes involved in liver fibrosis, including Col1a1, Acta2, Tgfb1, and Mmp13, and hepatic inflammation, including
Tnfa, Illb, Il12b, Nos2, Ccl2, Ccl5, and Adgre1 (F4/80), was significantly elevated in the livers from Nrg4-null mice (Figure 2F). These findings illustrate that $\mathrm{Nrg} 4$ deficiency worsened liver inflammation and fibrosis following NASH diet feeding. Th17 cells 
A

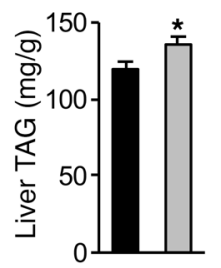

B

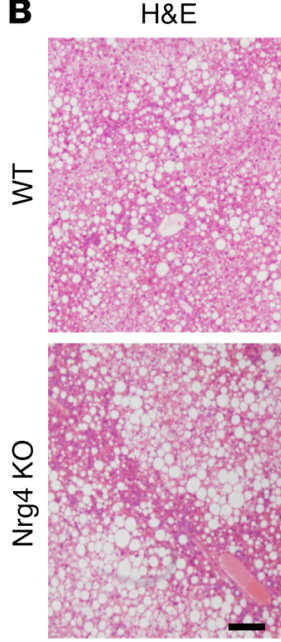

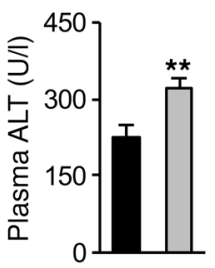

Sirius red
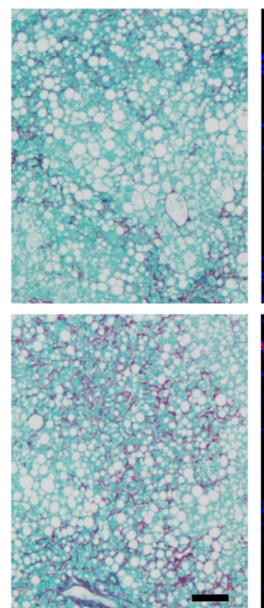

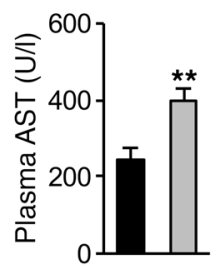

F4/80 \& DAPI
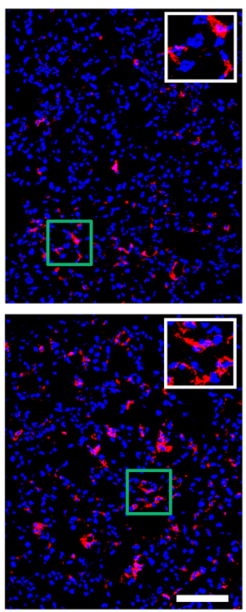

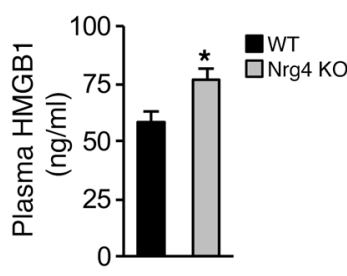

D

Cleaved-CASP3

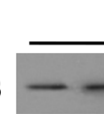

WT

$\mathrm{Nrg} 4 \mathrm{KO}$
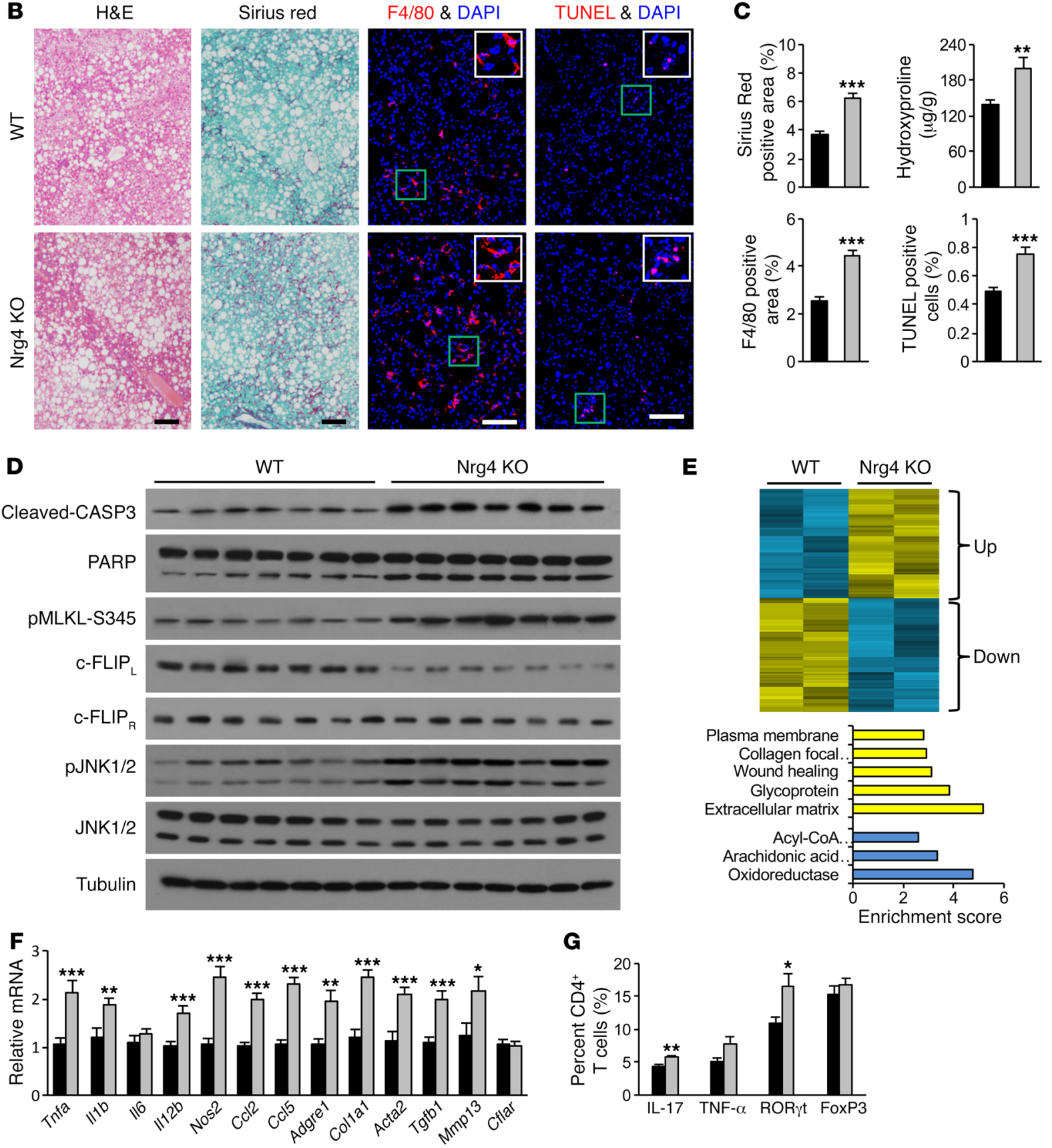

G

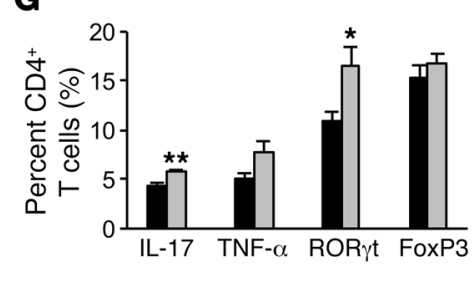

Figure 2. Nrg4 deficiency exacerbates diet-induced NASH in mice. Control WT ( $n=12$, black) and Nrg4-KO ( $n=12$, gray) male mice were fed NASH diet for 20 weeks, starting at 3 months of age. (A) Plasma ALT, AST, and HMGB1 levels and liver TAG content. Data represent mean $\pm S E M$. ${ }^{*} P<0.05 ;{ }^{* *} P<0.01$, 2-tailed unpaired Student's $t$ test. (B) H\&E, sirius red, F4/80 immunofluorescence, and TUNEL staining of liver sections. Scale bars: $100 \mu \mathrm{m}$. (C) Quantification of sirius red, F4/80, and TUNEL staining images and liver hydroxyproline content. Data represent mean $\pm \mathrm{SEM}$. ${ }^{* *} P<0.01$; ${ }^{* * *} P<0.001,2$-tailed unpaired Student's $t$ test. (D) Immunoblots of total liver lysates from NASH diet-fed mice. (E) Heatmap representation of hepatic genes up- or downregulated by Nrg4 deficiency following NASH diet feeding (top). Enrichment of biological processes in these 2 clusters (bottom). (F) qPCR analysis of hepatic gene expression. Data represent mean $\pm \mathrm{SEM}$. ${ }^{*} P<0.05 ;{ }^{*} P<0.01 ;{ }^{* *} P<0.001$, 2-tailed unpaired Student's $t$ test. (G) Flow cytometry analysis of liver $\mathrm{CD}^{+} \mathrm{T}$ cells. Results are expressed as percentage (\%) of IL-17+, TNF- $\alpha^{+}, \mathrm{ROR} \gamma \mathrm{t}^{+}$, and FOXP3 ${ }^{+}$cells in total liver CD4 ${ }^{+} \mathrm{T}$ cells. Data represent mean $\pm \mathrm{SEM}$. ${ }^{*} P<0.05 ;{ }^{* *} P<0.01,2$-tailed unpaired Student's $t$ test. 
and IL-17 have recently been implicated in promoting steatohepatitis and liver fibrosis (35). Flow cytometry analysis indicated that the populations of Th17 cells and ROR $\gamma \mathrm{t}^{+} \mathrm{CD} 4^{+} \mathrm{T}$ cells were significantly increased in Nrg4-null mouse livers (Figure 2G). These results suggest that increased Th17 cell infiltration and IL-17 signaling may contribute to the exacerbation of NASH phenotypes in Nrg4-null mice. Together, these data demonstrate that Nrg4 signaling protects mice from diet-induced NASH and that its deficiency exacerbates key pathogenic events of NASH.

$\mathrm{Tg}$ restoration of the $\mathrm{Nrg} 4$ endocrine axis protects mice from $\mathrm{NASH}$. Having established that Nrg4 deficiency is causally linked to NASH, we next explored whether Tg restoration of Nrg4 signaling is sufficient to ameliorate diet-induced NASH in mice. We fed WT and fat-specific Nrg4-Tg mice standard chow or NASH diet for approximately 20 weeks. Hepatic steatosis and fibrosis, plasma ALT and AST, and mRNA expression of genes involved in liver fibrosis and inflammation were similar between 2 groups following chow feeding (Supplemental Figure 3, A-D). Following NASH diet feeding, Nrg4-Tg mice had moderately lower liver fat content (Figure 3A), slightly smaller lipid droplets in hepatocytes (Supplemental Figure 3, E and F), and significantly lower plasma ALT, AST, and HMGB1 levels (Figure 3A), suggesting that Tg expression of Nrg4 may improve NASH diet-induced liver injury. In support of this, liver fibrosis and hydroxyproline content were significantly reduced in Tg mouse livers (Figure 3, B and C). Immunofluorescence staining revealed that the abundance of $\mathrm{F} 4 / 80^{+}$macrophage and TUNEL-positive cells was reduced in Tg mice, indicative of attenuated liver inflammation and hepatocyte death. Consistently, mRNA expression of genes involved in hepatic inflammation and fibrosis was significantly downregulated in Nrg4-Tg mouse livers, accompanied by reduced population of Th17 cells and ROR $\gamma \mathrm{t}^{+} \mathrm{CD} 4^{+}$ $\mathrm{T}$ cells (Figure 3, D and E). Nrg4-Tg mouse livers exhibited reduced CASP3 and PARP cleavage and MLKL phosphorylation (Figure 3F). The levels of phosphorylated JNK were lower in $\mathrm{Tg}$ livers, whereas c-FLIP ${ }_{L}$ protein levels were elevated in the livers of Nrg4-Tg mice. The strikingly opposite effects of Nrg4 deficiency and Tg expression on diet-induced NASH illustrate a crucial role of Nrg4 in serving as an endocrine checkpoint for steatosisto-NASH progression. Given that Nrg4 manipulations only moderately affect the severity of hepatic steatosis, our results suggest that Nrg4 may exert cytoprotective functions on hepatocytes independently of its regulation of lipid metabolism.

Nrg4 signaling protects hepatocytes from stress-induced cell death through $c$-FLIP ${ }_{L}$. Hepatocyte death is at the center of NASH initiation and progression, triggering chronic inflammation and fibrosis in the liver. The findings that liver injury was increased in Nrg4-null mice while attenuated in Nrg4-Tg mice prompted us to investigate whether Nrg4 directly modulates hepatocyte death. Previous work has demonstrated that liver expresses all ErbB receptors except ErbB2 (36). However, ErbB4 expression in primary hepatocyte culture is relatively low. We reconstituted $\mathrm{Nrg} 4 / \mathrm{ErbB} 4$ signaling by transducing primary hepatocytes with a recombinant adenoviral vector expressing ErbB4, as previously described (25). As expected, recombinant Nrg4 dose-dependently stimulated ErbB4 receptor tyrosine phosphorylation and AKT phosphorylation in transduced primary hepatocytes (Figure 4A). $\mathrm{NASH}$-associated hepatocyte death results from an array of stress signals, most notably lipotoxicity and proinflammatory cytokines. Previous studies have demonstrated that treatments of primary hepatocytes with a combination of palmitic acid (PA) and TNF- $\alpha$ $(\mathrm{PA} / \mathrm{TNF}-\alpha)$ robustly triggered cell death that mimics NASHinduced liver injury $(21,37)$. To investigate whether Nrg4 plays a direct role in the regulation of hepatocyte death, we treated primary hepatocytes transduced with GFP or ErbB4 adenovirus with PA/TNF- $\alpha$. As expected, PA/TNF- $\alpha$ treatment increased CASP3 and PARP cleavage and MLKL phosphorylation in hepatocytes transduced with Ad-GFP, indicative of induction of apoptotic and necroptotic cell death in response to PA and TNF- $\alpha$ (Figure 4B). $\mathrm{Nrg} 4$ treatment strongly attenuated cell death markers in primary hepatocytes expressing ErbB4. We measured the release of lactate dehydrogenase (LDH) into culture media as an indicator of hepatocyte death (38). LDH release by primary hepatocytes was markedly increased in response to PA/TNF- $\alpha$ treatment (Figure $4 \mathrm{C}$ ). Activation of $\mathrm{Nrg} 4$ signaling strongly attenuated $\mathrm{LDH}$ release induced by PA/TNF- $\alpha$. Further, flow cytometry analysis using annexin $\mathrm{V}$ and propidium iodide (PI) costaining indicated that PA/ TNF- $\alpha$ treatment increased the frequency of apoptotic (annexin V positive and PI negative) and dead (double positive) cells, which was significantly alleviated by Nrg4 (Figure 4, D and E). Similar protective effects of $\mathrm{Nrg} 4$ were also observed in transduced Hepa 1 hepatoma cells stably expressing ErbB4 (Figure 4, F and G, and Supplemental Figure 4, A-C).

c-FLIP ${ }_{L}$ is a cell death inhibitory protein that has been shown to suppress both apoptosis and necroptosis and protect against liver injury in mice (39-41). As shown above, c-FLIP ${ }_{\mathrm{L}}$ protein level was reduced in Nrg4-null mouse livers, but elevated by Nrg4$\mathrm{Tg}$ expression in adipose tissue. These observations suggest that c-FLIP ${ }_{\text {L }}$ may be a downstream effector of Nrg4 that links Nrg4/ ErbB4 signaling to hepatocyte death. Interestingly, c-FLIP mRNA expression in Nrg4-null and - Tg mouse livers remained largely unchanged (Figure 2F and Figure 3D), suggesting that Nrg4 may regulate $\mathrm{c}-\mathrm{FLIP}_{\mathrm{L}}$ protein levels through posttranslational mechanisms. In support of this, we found that endogenous $C-F L I P{ }_{L}$ protein levels were reduced in hepatoma cells in response to PA/ TNF- $\alpha$ treatment without affecting $c$-Flip mRNA levels (Figure 5A and Supplemental Figure 5A). Nrg4 treatment stimulated AKT phosphorylation, attenuated JNK phosphorylation, and nearly completely blocked the decrease of $\mathrm{c}-$ FLIP $_{\mathrm{L}}$ protein expression by $\mathrm{PA} / \mathrm{TNF}-\alpha$ treatment. Cycloheximide (CHX) chase studies indicated that $\mathrm{Nrg} 4$ increased the half-life of $\mathrm{c}-\mathrm{FLIP}_{\mathrm{L}}$ in hepatoma cells (Figure 5B). Accordingly, the proteasome inhibitor MG132 blocked PA/TNF- $\alpha$-induced decrease of c-FLIP ${ }_{\mathrm{L}}$ (Figure $5 \mathrm{C}$ ), consistent with a posttranslational mechanism of regulation.

We next performed studies in Hepa 1 cells stably expressing

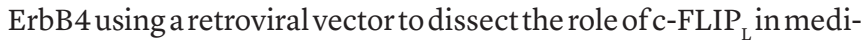
ating the cytoprotective activity of Nrg4. Interestingly, c-FLIP ${ }_{L}$ protein level was markedly reduced, whereas CASP3 cleavage and MLKL phosphorylation were stimulated, in response to PA/TNF- $\alpha$ treatment (Figure 5D). The effects of PA/TNF- $\alpha$ on c-FLIP $\mathrm{L}_{\text {pro- }}$ tein level and cell death markers were partially reversed by Nrg4 treatment. Further, adenovirus-mediated expression of $\mathrm{c}-\mathrm{FLIP}_{\mathrm{L}}$ greatly diminished PA/TNF- $\alpha$-induced apoptotic and necroptotic cell death. These results were confirmed by LDH release and flow cytometry analyses (Figure 5, E and F, and Supplemental Figure 
A
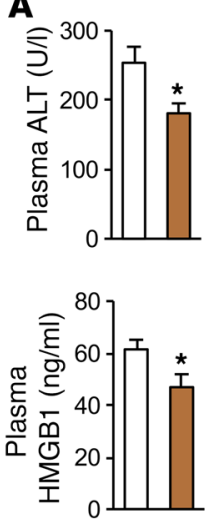
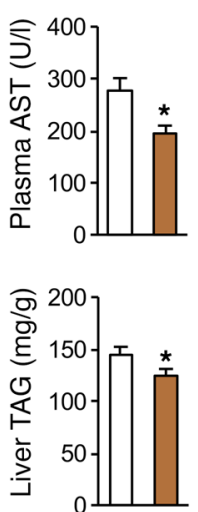

B

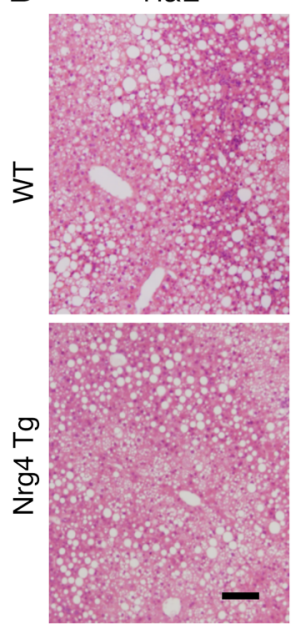

Sirius Red
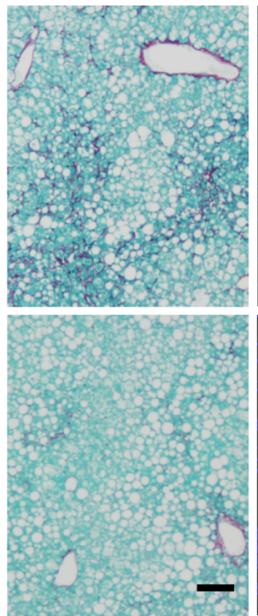

F4/80 \& DAPI
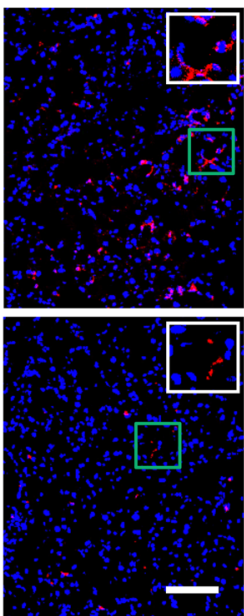

TUNEL \& DAPI
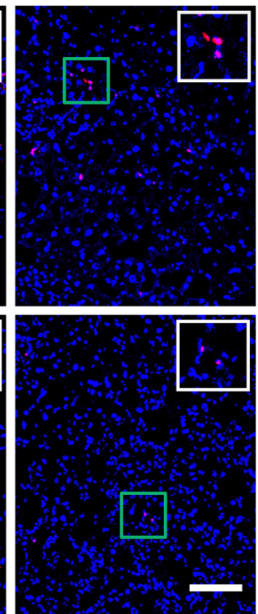
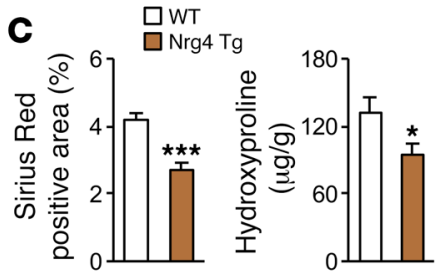

$F$

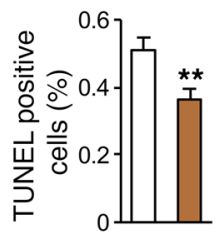

Cleaved-CASP3

PARP

pMLKL-S345

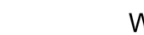

WT

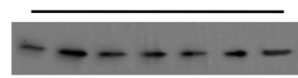

\section{c}

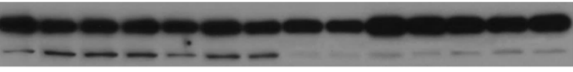

D
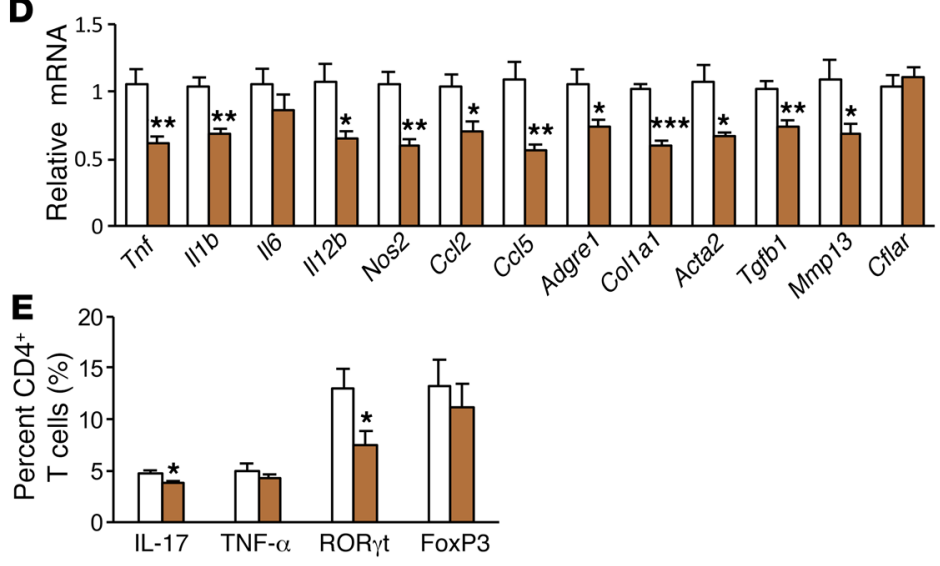

Figure 3. Tg restoration of the Nrg4 endocrine axis protects mice from diet-induced NASH. Control WT ( $n=13$, white) and Nrg4-Tg ( $n=9$, brown) male mice were fed a NASH diet for 20 weeks, starting at 3 months of age. (A) Plasma ALT, AST, and HMGB1 levels and liver TAG content. Data represent mean \pm SEM. ${ }^{*} P<0.05$, 2-tailed unpaired Student's $t$ test. (B) H\&E, sirius red, F4/80 immunofluorescence, and TUNEL staining of liver sections. Scale bars: 100 $\mu \mathrm{m}$. (C) Quantification of the sirius red, F4/80, and TUNEL staining images and liver hydroxyproline content. Data represent mean \pm SEM. ${ }^{*} P<0.05$; ${ }^{* *} P<0.01$; ${ }^{* *} P<0.001$, 2-tailed unpaired Student's $t$ test. (D) qPCR analysis of hepatic gene expression. Data represent mean $\pm S E M$. ${ }^{*} P<0.05$; ${ }^{* *} P<0.01 ;{ }^{* *} P<0.001,2$-tailed unpaired Student's $t$ test. (E) Flow cytometry analysis of liver CD4+ $T$ cells. Results are expressed as percentage (\%) of $\mathrm{IL}-17^{+}, \mathrm{TNF}-\alpha^{+}, \mathrm{ROR} \gamma \mathrm{t}^{+}$, and $\mathrm{FOXP3}{ }^{+}$cells in total liver CD4 ${ }^{+}$T cells. Data represent mean $\pm \mathrm{SEM} .{ }^{*} P<0.05,2$-tailed unpaired Student's $t$ test. (F) Immunoblots of total liver lysates from NASH diet-fed mice.

4D). We did not observe further improvement of cell viability by

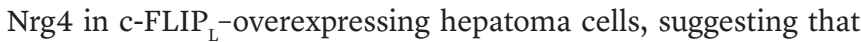
the protective effects of $\mathrm{Nrg} 4$ are largely mediated by its induction of $\mathrm{c}-\mathrm{FLIP}_{\mathrm{L}}$ protein expression.

Nrg4 attenuates ubiquitination and proteasomal degradation of $c-F L I P_{L}$. We performed ubiquitination assay to determine whether PA/TNF- $\alpha$ and Nrg4 play an antagonistic role in c-FLIP ${ }_{L}$ ubiquitination. As shown in Figure 6A, PA/TNF- $\alpha$ treatment induced robust polyubiquitination of $\mathrm{c}-\mathrm{FLIP}_{\mathrm{L}}$ that was largely reversed by $\mathrm{Nrg} 4$ treatment. We next determined whether AKT activation by $\mathrm{Nrg} 4$ is required for its regulation of $\mathrm{c}-\mathrm{FLIP}_{\mathrm{L}}$ protein stability. We performed PA/TNF- $\alpha$ and Nrg4 treatments without or with AKTi, a specific AKT inhibitor. The ability of Nrg4 to attenuate c-FLIP ${ }_{L}$ ubiquitination in response to $\mathrm{PA} / \mathrm{TNF}-\alpha$ treatment was greatly diminished in the presence of AKTi (Figure 6B). As a result, Nrg4 failed to stabilize c-FLIP ${ }_{L}$ protein and suppress PA/TNF- $\alpha-$ induced cell death, as shown by CASP3 cleavage, MLKL phosphorylation, and $\mathrm{LDH}$ release (Figure 6, B-D). On the contrary, inhibition of JNK kinase activity blocked the induction of c-FLIP ubiquitination by $\mathrm{PA} / \mathrm{TNF}-\alpha$ treatment, leading to improved cell viability (Figure 6, E and F). Nrg4 treatment did not further

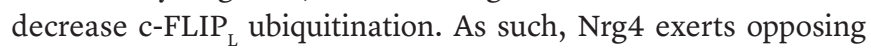


A

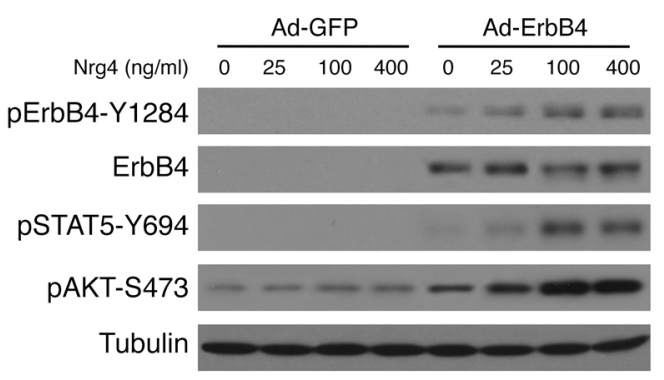

B
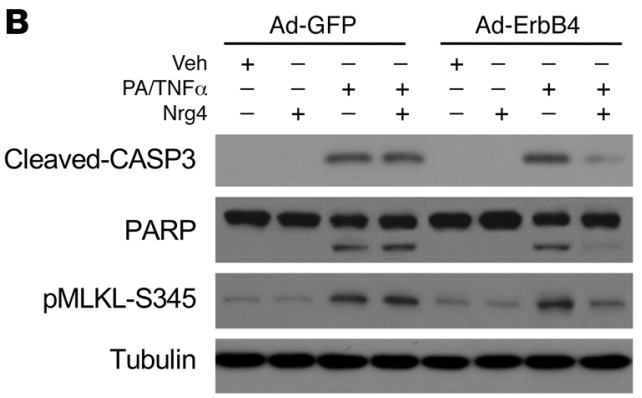

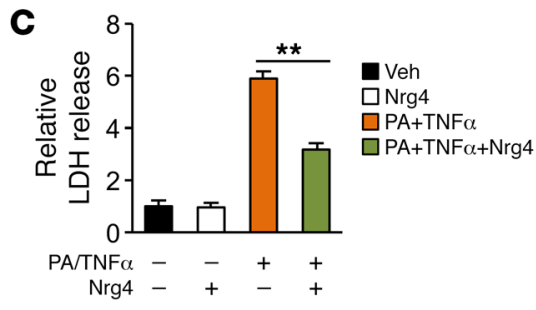

E

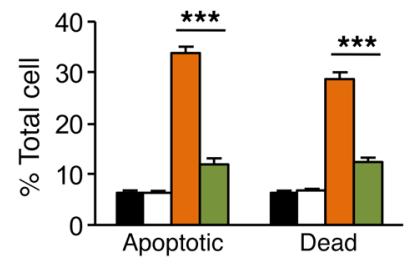

D

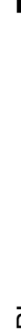

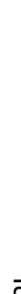

$\bar{n}$

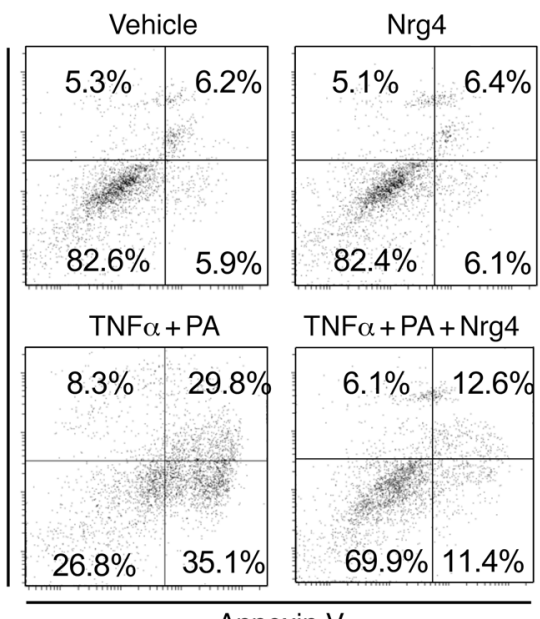

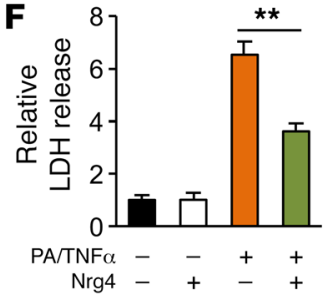

G

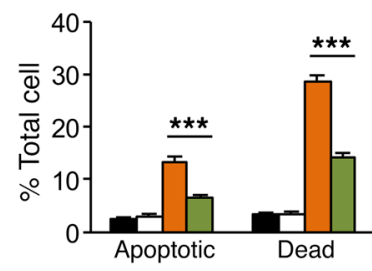

Figure 4. Nrg4 signaling protects hepatocytes from stress-induced cell death. (A) Immunoblots of total lysates from primary hepatocytes transduced with GFP or ErbB4 adenovirus and treated with Nrg4 for 20 minutes. (B) Immunoblots of total lysates from primary hepatocytes transduced with GFP or ErbB4 adenovirus treated with $150 \mu \mathrm{M}$ PA for 2 hours followed by addition of $40 \mathrm{ng} / \mathrm{ml} \mathrm{TNF}-\alpha$ (PA/TNF- $\alpha$ ) and $100 \mathrm{ng} / \mathrm{ml} \mathrm{Nrg} 4$ for 20 hours. (C) LDH activity in culture media from hepatocytes transduced with Ad-ErbB4 and treated as indicated for 20 hours. Data represent mean \pm SEM. ${ }^{* *} P<0.01,1$-way ANOVA. (D) Flow cytometry analysis of hepatocytes (20 hours treatment) following annexin $V$ and PI staining. Double-positive cells are considered dead, whereas annexin V-positive and PI-negative cells are apoptotic. (E) Quantitation of hepatocyte cell death based on annexin V/PI staining. Data represent mean \pm SEM. ${ }^{* *} P<0.001$, 1-way ANOVA. (F) LDH activity in culture media from Hepa 1 cells stably expressing ErbB4 and treated as indicated for 20 hours. Data represent mean \pm SEM. ${ }^{* *} P<0.01$, 1-way ANOVA. (G) Flow cytometry analysis of cell death in treated Hepa 1 cells stably expressing ErbB4 (20 hours treatment). Data represent mean \pm SEM. ${ }^{* *} P<0.001,1$-way ANOVA.

effects on the AKT and JNK signaling pathways to regulate c-FLIP ubiquitination and proteasomal degradation in response to PA/ TNF- $\alpha$. Further, the reciprocal regulation of c-FLIP protein levels in Nrg4-KO and -Tg mouse livers likely reflects cell-autonomous action of $\mathrm{Nrg} 4$ in hepatocytes.

Previous work has identified Itchy E3 ubiquitin protein ligase

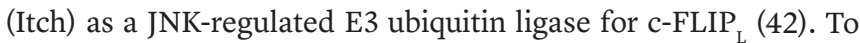
investigate whether Itch is required for ubiquitination and proteasomal degradation of c-FLIP ${ }_{\mathrm{L}}$ in response to PA/TNF- $\alpha$, we knocked down Itch in Hepa 1 cells using 2 independent siRNA pools against Itch. Surprisingly, siRNA knockdown of Itch did not appear to affect c-FLIP $\mathrm{L}_{\mathrm{L}}$ ubiquitination and degradation and PA/TNF- $\alpha$-induced cell death (Supplemental Figure 5, B and C). These results suggest that it is unlikely that Itch is the E3 ubiquitin ligase responsible for PA/TNF- $\alpha$-induced c-FLIP ${ }_{\mathrm{L}}$ ubiquitination.

Restoration of hepatic c-FLIP expression blocks steatosis-toNASH progression due to Nrg4 deficiency. The above studies illustrate the nature of molecular signaling that links Nrg4 signaling to regulation of C-FLIP ${ }_{L}$ protein stability and hepatocyte cell death. A key prediction of this model is that restoration of hepatic c-FLIP protein expression will improve diet-induced NASH in mice due to $\mathrm{Nrg} 4$ deficiency. To test this, we generated a c-FLIP ${ }_{\mathrm{L}}$ expression vector using adeno-associated virus (AAV) type 8 under the control of the liver-specific thyroid hormone-binding globulin $(\mathrm{T} b g)$ promoter. Tail vein injection of AAV-c-FLIP $P_{L}$ elevated c-FLIP expression specifically in the liver, but not other tissues, such as adipose tissue, spleen, and lung (Supplemental Figure 6A). We fed WT and Nrg4-null mice NASH diet for 8 weeks and transduced these mice with control AAV-GFP or AAV-c-FLIP ${ }_{\mathrm{L}}$ virus. Four treatment groups were obtained: WT mice injected with AAVGFP (WT/GFP) or AAV-c-FLIP ${ }_{\text {L }}\left(\mathrm{WT} / \mathrm{c}-\mathrm{FLIP}_{\mathrm{L}}\right.$ ) and Nrg4-KO mice injected with AAV-GFP (Nrg4 KO/GFP) or AAV-c-FLIP ${ }_{\mathrm{L}}$ (Nrg4 $\mathrm{KO} / \mathrm{c}-\mathrm{FLIP}_{\mathrm{L}}$ ). We examined NASH features in transduced mice following an additional 12 weeks of NASH diet feeding. Similar to what occurred in the studies shown in Figure 2, Nrg4 deficiency accelerated diet-induced NASH in mice. Compared with WT/GFP, Nrg4-KO/GFP mice had higher plasma ALT, AST, and HMGB1 levels, developed more severe liver fibrosis, and exhibited increased presence of apoptotic cells (Figure 7, A-C). While AAV-mediated overexpression of c-FLIP $\mathrm{L}_{\mathrm{L}}$ moderately improved 
A
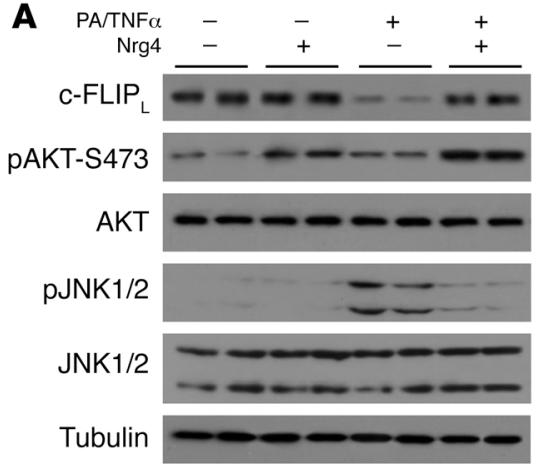

C $\mathrm{PA} / \mathrm{TNF} \alpha-+++++$

\begin{tabular}{|c|c|c|c|c|c|c|}
\hline PA/TNF $\alpha$ & - & - & + & + & + & + \\
\hline Nrg4 & - & + & - & + & - & + \\
\hline MG132 & - & - & - & - & + & + \\
\hline$c-F L I P_{L}$ & 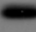 & - & - & - & - & - \\
\hline
\end{tabular}

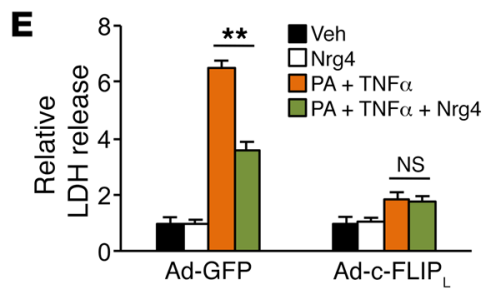

B
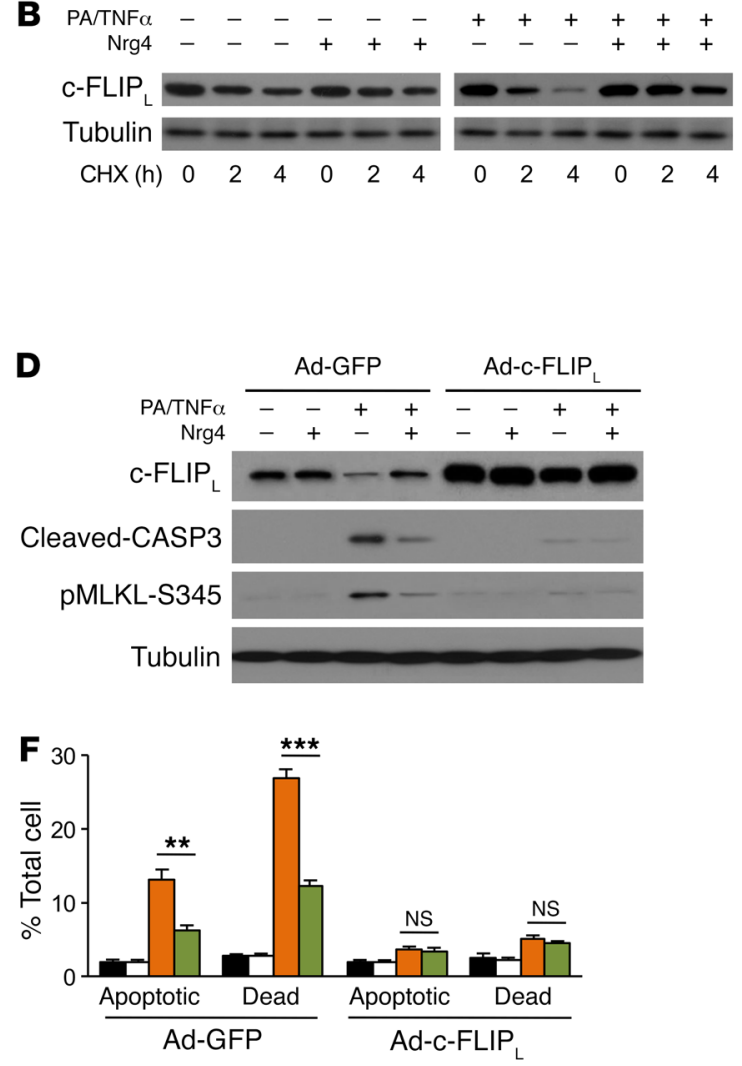

Figure 5. Stabilization of c-FLIP ${ }_{L}$ by Nrg4 attenuates stress-induced cell death in hepatoma cells. The following experiments were performed in Hepa 1 cells stably expressing ErbB4. Cells were cultured in serum-free medium during treatment. (A) Immunoblots of total lysates from cells treated with PA/TNF- $\alpha$ in the absence or presence of $100 \mathrm{ng} / \mathrm{ml} \mathrm{Nrg4}$ for 6 hours. (B) Immunoblots of total lysates from cells treated with PA/TNF- $\alpha$ without or with $100 \mathrm{ng} / \mathrm{ml} \mathrm{Nrg} 4$ and chased for different times in the presence of $2 \mu \mathrm{M}$ CHX. (C) Immunoblots of total lysates from cells treated with PA/TNF- $\alpha$ without or with $100 \mathrm{ng} / \mathrm{ml} \mathrm{Nrg} 4$ and chased for 6 hours in the presence of $10 \mu \mathrm{M}$ MC132. (D) Immunoblots of total lysates from treated cells. Hepa 1 cells stably expressing ErbB4 were transduced with Ad-GFP or Ad-c-FLIP adenoviral vector. Transduced cells were treated with $100 \mu \mathrm{M}$ PA for 2 hours followed by addition of $20 \mathrm{ng} / \mathrm{ml} \mathrm{TNF}-\alpha$ and $100 \mathrm{ng} / \mathrm{ml} \mathrm{Nrg} 4$ for 20 hours. (E) LDH release by treated Hepa 1 cells (20 hours treatment). Data represent mean \pm SEM. ${ }^{* *} P<0.01,1$-way ANOVA. (F) Flow cytometry analysis (20 hours treatment). Data represent mean \pm SEM. ${ }^{* *} P<0.01$; ${ }^{* *} P<0.001,1$-way ANOVA.

NASH parameters in WT mice, it nearly completely eliminated worsening of NASH in Nrg4-null mice. Hepatic gene expression analyses indicated that the expression of genes involved in inflammation and liver fibrosis in Nrg4-null mice was greatly attenuated by AAV-c-FLIP ${ }_{L}$, reaching levels similar to those in WT mice (Figure 8A). Accordingly, CASP3 and PARP cleavage and MLKL phosphorylation in Nrg4-KO mice also returned to control levels following transduction with AAV-c-FLIP ${ }_{\mathrm{L}}$ (Figure 8B). Liver fat content and hepatic lipid droplet number and size, however, were not significantly affected by AAV-mediated overexpression of c-FLIP ${ }_{\mathrm{L}}$ (Supplemental Figure 6, B-D). These results support a critically important functional role of c-FLIP ${ }_{\mathrm{L}}$ regulation by $\mathrm{Nrg} 4$ in mediating its effects on NASH progression.

\section{Discussion}

A hallmark of NASH is the presence of persistent liver injury as a result of increased hepatocyte death, which serves as a pathogenic trigger for liver inflammation and fibrosis $(13,43)$. Genetic manipulations that impinge on the cell death pathways, includ- ing apoptosis and necroptosis, exert strong effects on the progression of both acute and chronic liver disease. Despite this, the signaling mechanisms that counter pathophysiological insults and preserve hepatocyte health in NASH remain poorly understood. In this study, we uncovered an adipose-hepatic endocrine axis that functions as a checkpoint for diet-induced NASH in mice. Nrg4 protects hepatocytes from apoptosis and necroptosis triggered by metabolic and inflammatory stress by attenuating ubiquitination and proteasomal degradation of c-FLIP (Figure 8C). As such, activation of Nrg4-mediated endocrine signaling may provide a new therapeutic avenue for halting and/or reversing NASH pathogenesis in humans.

The observation that $\mathrm{Nrg} 4$, a fat-derived endocrine factor, plays a direct role in cell death signaling in hepatocytes came somewhat as a surprise. We previously demonstrated that Nrg4 exerts pleiotropic effects on hepatic and systemic energy metabolism in diet-induced obesity (25). However, HFD used in the previous studies was inadequate in triggering liver injury and NASH. Here, we used a high-fat, high-fructose diet that elicits key aspects of human NASH pathologies in mice (34). Notably, NASH dietinduced apoptotic and necroptotic cell death in mouse liver was linked to downregulation of C-FLIP , a prosurvival factor, closely resembling the observations in NASH patient livers. Several lines of evidence support the pathophysiological significance of $\mathrm{Nrg} 4$ in gating the transition from hepatic steatosis to NASH. Nrg4-null mice developed more severe liver injury upon NASH diet feeding, as shown by elevated plasma levels of ALT, AST, and HMGB1. Liver cell death and inflammation were increased in mice lacking $\mathrm{Nrg} 4$, leading to more severe liver fibrosis. In contrast, fat-specific $\mathrm{Tg} \mathrm{Nrg} 4$ expression restored Nrg4 deficiency and ameliorated the severity of diet-induced NASH in mice. Activation of $\mathrm{Nrg} 4$ signaling attenuated apoptotic and necroptotic cell death in hepatocytes in a cell-autonomous manner, illustrating a direct role of $\mathrm{Nrg} 4$ in preserving liver health under stress conditions.

At the mechanistic level, Nrg4 engages the prosurvival factor c-FLIP ${ }_{\mathrm{L}}$ to attenuate cell death. Two isoforms of c-FLIP protein have been identified in mice: c-FLIP ${ }_{\mathrm{R}}$ and $\mathrm{c}-\operatorname{FLIP}_{\mathrm{L}}(31,32)$. 
A

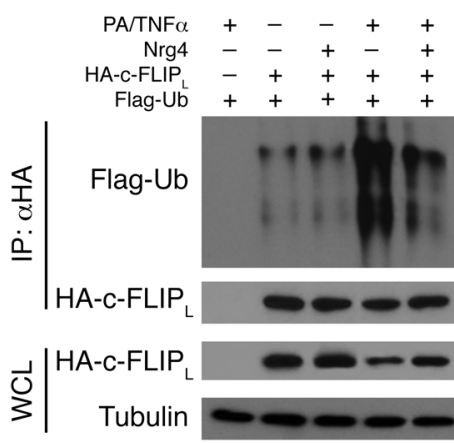

C

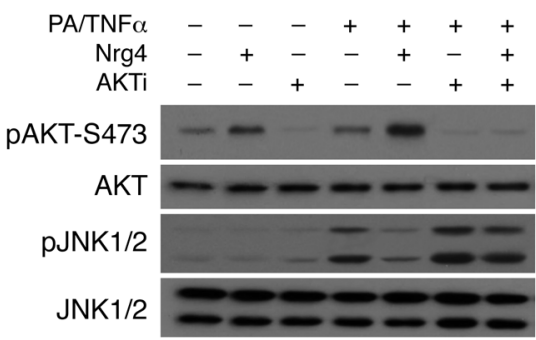

$\mathbf{E}$

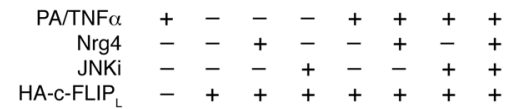

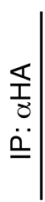

HA-C-FLIP

3

Cleaved-CASP3

pMLKL-S345

Tubulin
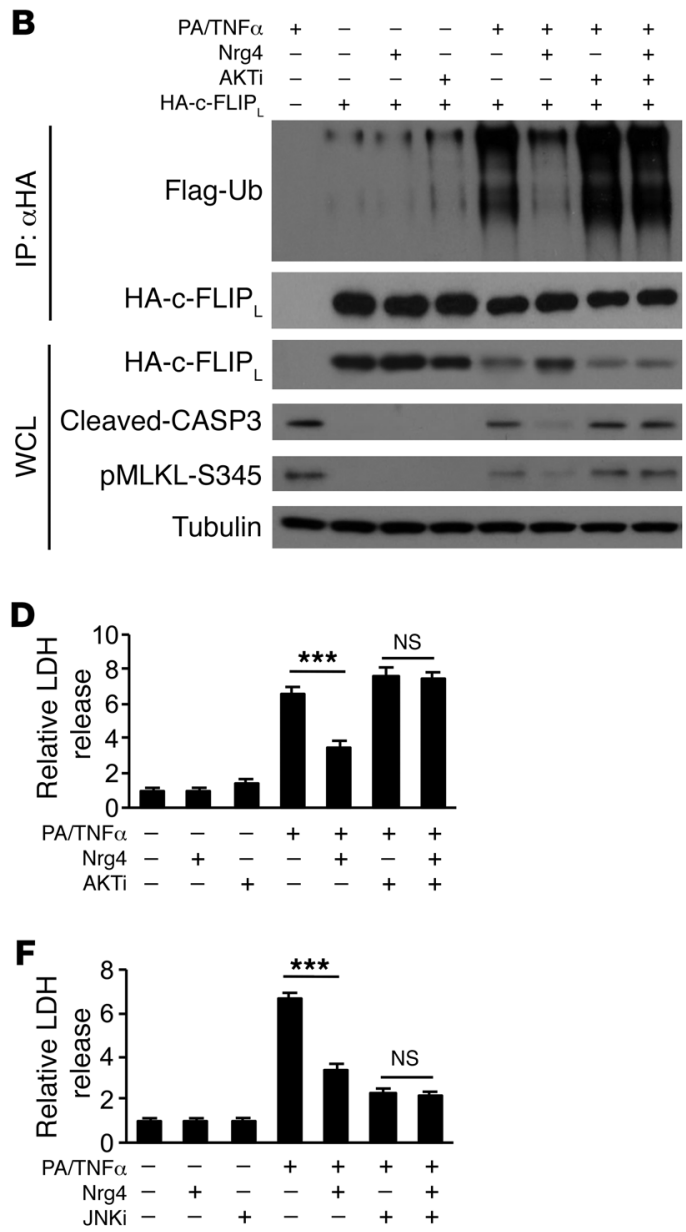

Figure 6. Nrg4 attenuates ubiquitination and proteasomal degradation of c-FLIP. The following experiments were performed in Hepa 1 cells stably expressing ErbB4. Cells were cultured in serum-free medium during treatment. WCL, whole cell lysates. (A) Immunoblots of IP and whole cell lysates from Hepa 1 cells transfected with plasmids encoding HA-tagged c-FLIP, and Flag-tagged ubiquitin (Flag-Ub) and treated with PA/TNF- $\alpha$ without or with $100 \mathrm{ng} / \mathrm{ml} \mathrm{Nrg} 4$ for 4 hours. (B) Immunoblots of IP and whole cell lysates from transfected Hepa 1 cells treated in the absence or presence of AKT kinase inhibitor $(20 \mu \mathrm{M})$ for 4 hours. (C) Immunoblots of total lysates from cells treated with PA/TNF- $\alpha$ without or with $100 \mathrm{ng} / \mathrm{ml} \mathrm{Nrg4}$ and in the absence or presence of AKT inhibitor $(20 \mu \mathrm{M})$ for 6 hours. (D) LDH release by Hepa 1 cells as treated for 20 hours. Data represent mean $\pm S E M$. ${ }^{* * *} P<0.001,1$-way ANOVA. (E) Immunoblots of IP and WCL from transfected Hepa 1 cells treated in the absence or presence of JNK1/2 kinase inhibitor (20 $\mu$ M) for 4 hours. (F) LDH release by Hepa 1 cells as treated for 20 hours. Data represent mean \pm SEM. ${ }^{* * *} P<0.001,1$-way ANOVA.

Interestingly, $\mathrm{Nrg} 4$ appeared to predominantly affect the c-FLIP isoform in the liver. c-FLIP forms a heterodimer with procaspase 8 to inhibit its activation and suppress the activation of apoptosis and necroptosis. For example, c-FLIP $\mathrm{L}_{\mathrm{L}}$ protects cells from Fas-induced cell death, involving both apoptosis and necroptosis (44), and the formation of cell death-inducing ripoptosome in response to Toll-like receptor 3 activation (45). Inactivation of c-FLIP ${ }_{\mathrm{L}}$ in mouse hepatocytes promotes liver injury triggered by death receptor agonists (39). A recent study demonstrated that AAV-mediated overexpression of c-FLIP ${ }_{\mathrm{L}}$ in the liver ameliorates NASH in mice and nonhuman primates (41), illustrating a crucial role of this factor in NASH. However, the physiological and pathophysiological cues that impinge on c-FLIP $\mathrm{L}$ remain largely unknown. Our current work uncovers adipose endocrine sig- naling via $\mathrm{Nrg} 4$ as a key regulator of c-FLIP $\mathrm{L}_{\mathrm{L}}$ ubiquitination and proteasome-mediated degradation. Nrg4 strongly blunts JNK1/2 activation in hepatocytes in an AKT-dependent manner following PA/TNF- $\alpha$ treatment, leading to increased c-FLIP ${ }_{\mathrm{L}}$ protein stability. These results are consistent with previous work that demonstrates an important role for JNK in posttranslational regulation of c-FLIP ${ }_{L}$ (42). Notably, restoration of hepatic c-FLIP ${ }_{L}$ protein expression alleviated diet-induced NASH in Nrg4-null mice without affecting steatosis, suggesting that the Nrg4/c-FLIP pathway exerts an independent effect on steatosis-to-NASH progression. Our work elucidates the molecular nature and pathophysiological significance of an endocrine checkpoint that regulates steatosisto-NASH progression. Future work will further validate Nrg4based therapeutic biologics for NASH treatment. 
A
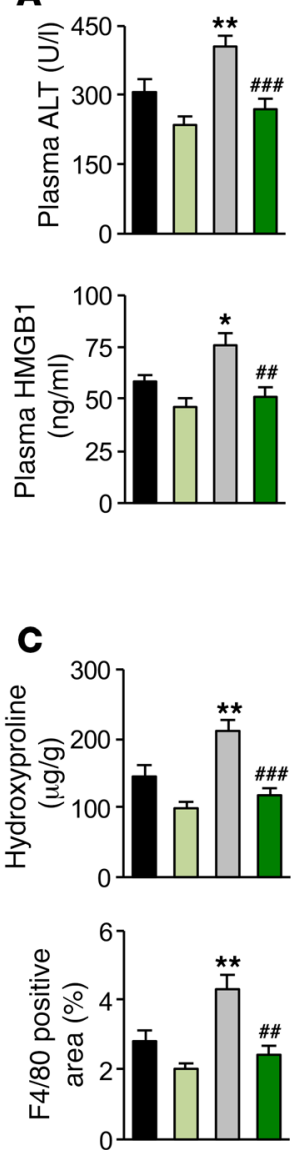

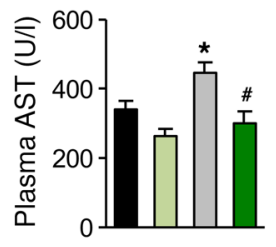

WT/GFP

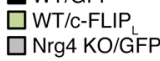

$\square \mathrm{Nrg} 4 \mathrm{KO} / \mathrm{C}-\mathrm{FLIP}$
B

\section{$\frac{1}{4}$}
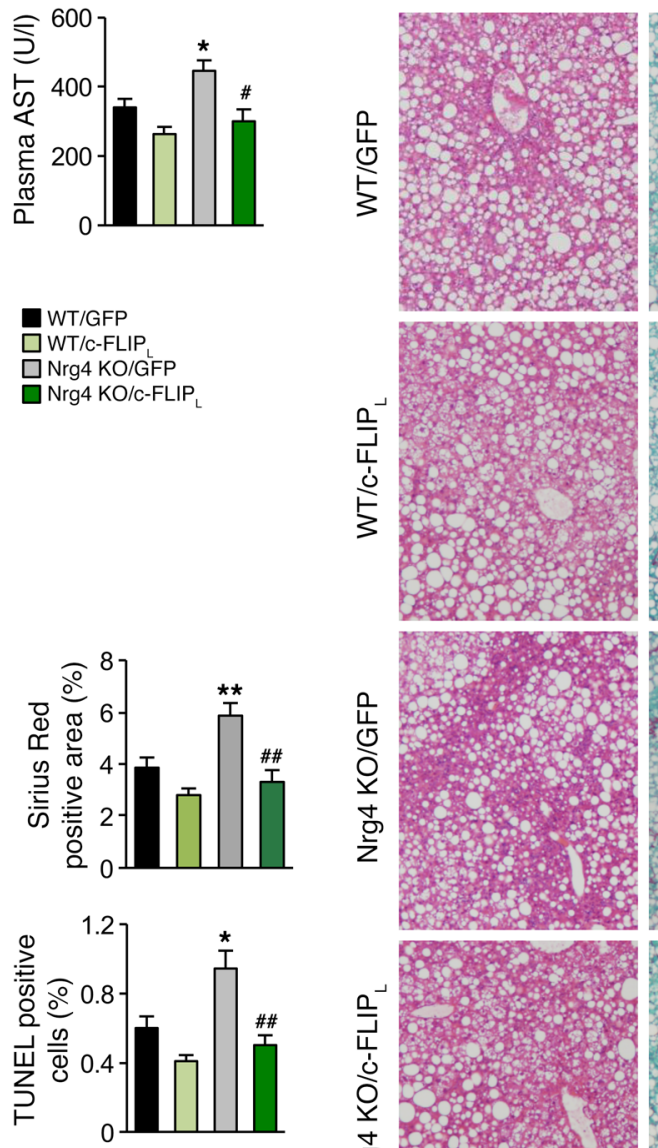
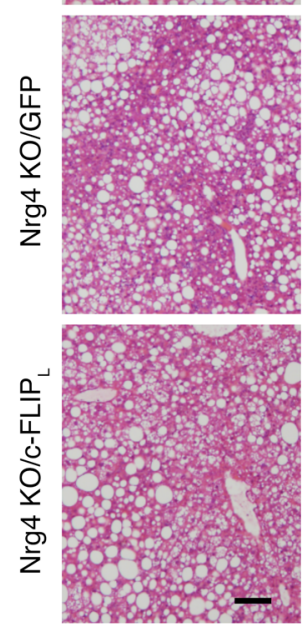

Sirius Red
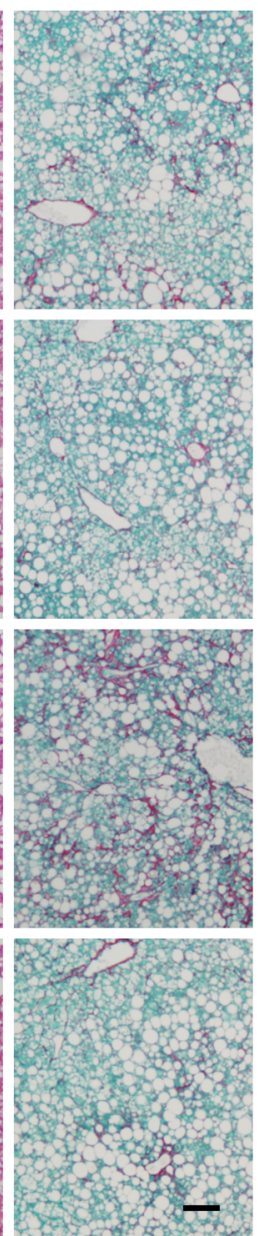

F4/80 \& DAPI
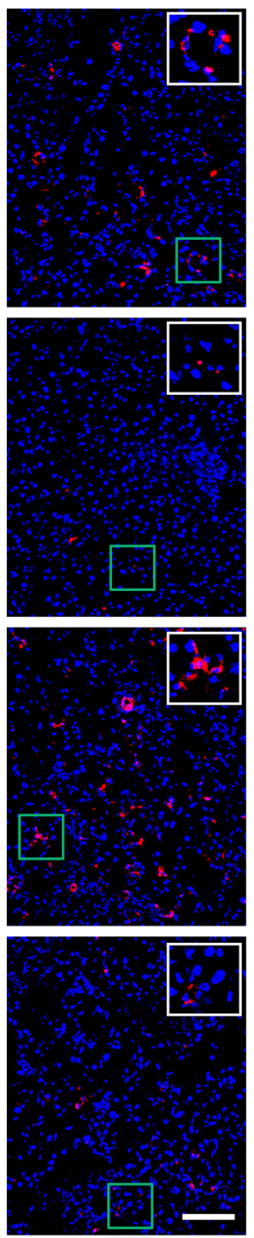

TUNEL \& DAPI
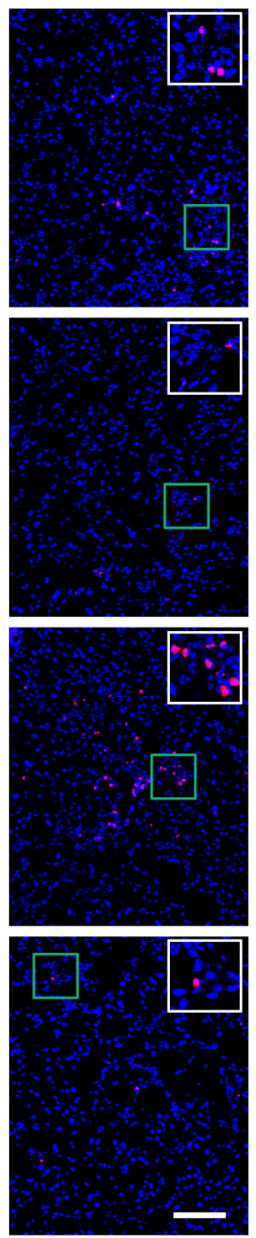

Figure 7. AAV-mediated restoration of hepatic c-FLIP alleviates NASH phenotype in Nrg4-deficient mice. The following parameters were measured in WT and Nrg4-KO male mice fed NASH diet for a total of 20 weeks. AAV8-GFP and AAV8-c-FLIP vectors were administered 8 weeks following the initiation of NASH feeding. WT/GFP group, $n=8$; WT/c-FLIP, group, $n=7$; Nrg4-KO/GFP group, $n=8$; Nrg4 KO/c-FLIP, group, $n=7$. (A) Plasma ALT, AST, and HMCB1 levels. Data represent mean \pm SEM. ${ }^{*} P<0.05,{ }^{* *} P<0.01$, WT/GFP vs. Nrg4-KO/GFP; ${ }^{\#} P<0.05$, \#\# $P<0.01$, \#\#\#<0.001, Nrg4-KO/GFP vs. Nrg4$\mathrm{KO} / \mathrm{C}-\mathrm{FLIP}_{1}$, 1-way ANOVA. (B) H\&E, sirius red, F4/80 immunofluorescence, and TUNEL staining of liver sections. Scale bars: $100 \mu \mathrm{m}$. (C) Quantification of sirius red, $\mathrm{F} 4 / 80$, and TUNEL staining images and liver hydroxyproline content. Data represent mean $\pm \mathrm{SEM}$. ${ }^{*} P<0.05$, ${ }^{*} P<0.01, \mathrm{WT} / \mathrm{GFP}$ vs. Nrg4 KO/ GFP; \#\#<0.01, \#\#P<0.001, Nrg4 KO/GFP vs. Nrg4 KO/c-FLIP, 1-way ANOVA.

\section{Methods}

Animal studies. Mice were housed under 12-hour light/12-hour dark cycles with free access to food and water. For chow feeding, mice were fed Teklad 5001 Laboratory Diet. For NASH diet feeding, mice were fed a diet containing $40 \%$ fat (of which $18 \%$ was trans fat), $22 \%$ fructose, and 2\% cholesterol (D09100301, Research Diets Inc.) starting at 3 months of age. Nrg4-knockout mice and Nrg4-Tg mice have been described previously (25). Nrg4-KO mice were purchased from the Mutant Mice Regional Resource Center (MMRRC) at UCD and backcrossed for more than 10 generations to the C57BL/6J background. Nrg4-Tg mice on a C57BL/6J background were generated at the Transgenic Animal Model Core at the University of Michigan. For AAV8 transduction, AAV8-GFP or AAV8-c-FLIP ${ }_{\mathrm{L}}\left(1 \times 10^{12}\right.$ genome copies/mouse $)$ was delivered by tail vein injection following 8 weeks of NASH diet feeding. After a total of 20 weeks of NASH diet feeding, mice were sacrificed for analysis.

We did not predetermine sample size, but used group sizes typical for this type of work in the literature. We randomly assigned mice of the same genotype to different treatments to minimize any potential bias. The investigators were not blinded to allocation during experiments and outcome assessment. We excluded mice that exhibited skin lesions due to fighting and growth retardation as a result of malocclusion.

Human studies. Human liver samples were obtained from the Liver Tissue Cell Distribution System at the University of Minnesota (Minneapolis, Minnesota, USA) (33). Both male and female individuals were included. The average ages for normal individuals and NASH patients were 55.8 and 52.5 years old, respectively. Individuals with an alcohol-drinking history ( 2 to 3 drinks/day) and liver cancer were excluded from the study.

Hepatocyte treatment and cell death studies. Primary hepatocytes were isolated as previously described by using collagenase type II from C57BL/6J mice (25). Hepatocytes were maintained in DMEM medium containing $10 \%$ bovine growth serum (BGS) at $37^{\circ} \mathrm{C}$ and $5 \% \mathrm{CO}_{2}$. Hepatocytes were infected with adenovirus on the same day as isolation. After 24 hours, cells were switched to serum-free DMEM for 12 hours 

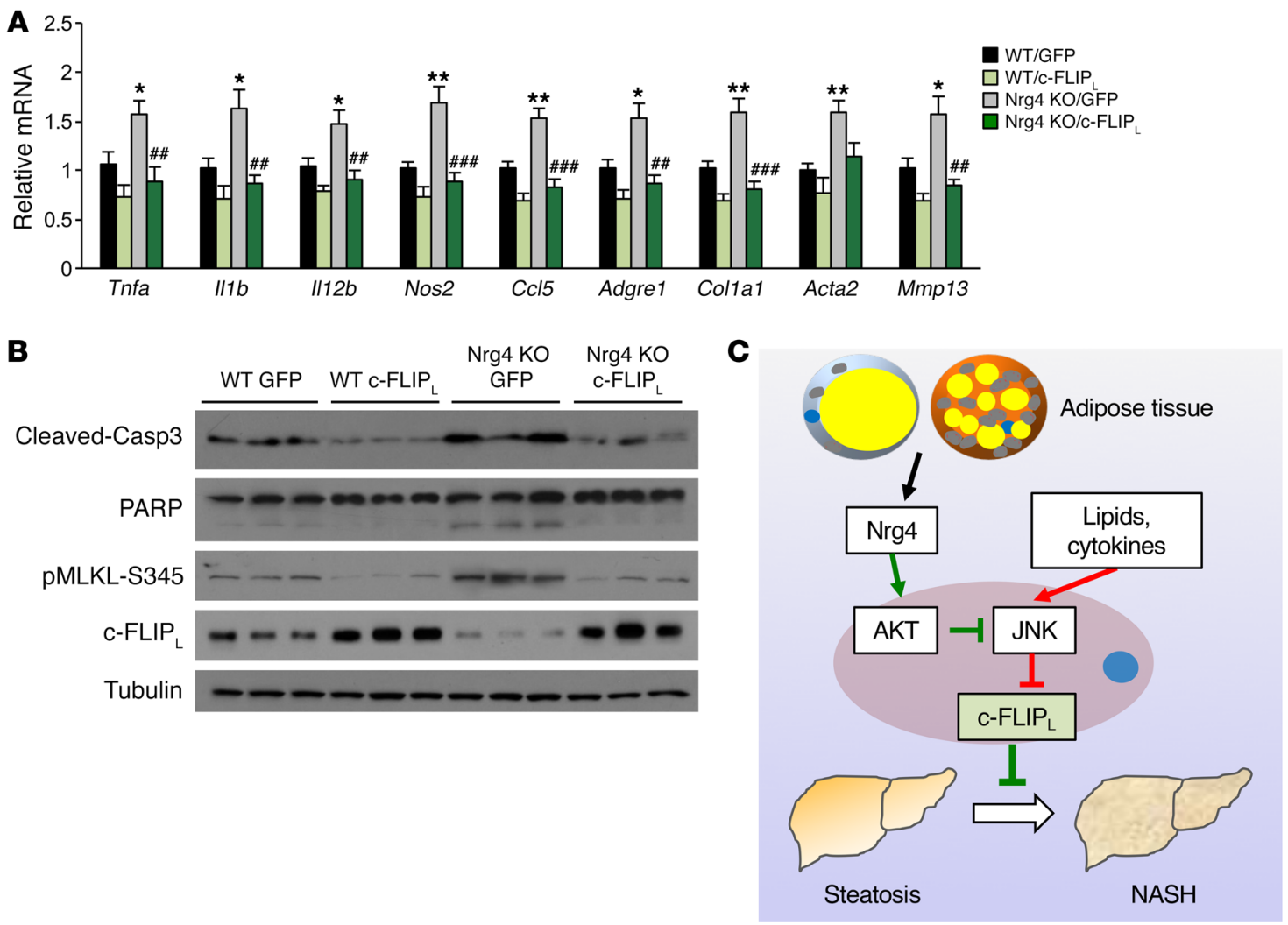

Figure 8. AAV-mediated restoration of hepatic c-FLIP, attenuates liver inflammation and cell death. The following parameters were measured in mice as described in the Figure 7 legend. (A) qPCR analysis of hepatic gene expression. Data represent mean $\pm \mathrm{SEM} .{ }^{*} P<0.05,{ }^{* *} P<0.01, \mathrm{WT} / \mathrm{GFP}$ vs. Nrg4 KO/ GFP; ${ }^{\#} P<0.01$, \#\# $P<0.001$, Nrg4 KO/GFP vs. Nrg4 KO/c-FLIP, 1-way ANOVA. (B) Immunoblots of total liver lysates. (C) Model depicting Nrg4-mediated endocrine signaling as a checkpoint for steatosis-to-NASH progression. Hepatic Nrg4 signaling counters c-FLIP, downregulation in response to lipotoxic and inflammatory stress, thereby preserving hepatocyte health and blocking the transition from hepatic steatosis to NASH.

before PA, TNF- $\alpha$, and/or Nrg4 treatment. Hepa 1 cells (ATCC, CRL1830) were maintained in DMEM medium containing $10 \% \mathrm{BGS}$ at $37^{\circ} \mathrm{C}$ and $5 \% \mathrm{CO}_{2}$. To establish a Hepa 1 cell line stably expressing ErbB4, Hepa 1 cells were infected with $\mathrm{pBABE}$ retroviral vector harboring the ErbB4 gene and screened with hygromycin $(250 \mu \mathrm{g} / \mathrm{ml}$ final concentration) for at least 2 weeks. Hepa 1 cells were switched o serum-free DMEM for 12 hours before treatments with PA, TNF- $\alpha$, AKT inhibitor (AKT Inhibitor VIII; Sigma-Aldrich, catalog A6730), JNK inhibitor (SP 600125; Sigma-Aldrich, catalog S5567), MG132 (Sigma-Aldrich, catalog 474787), and Nrg4 (Novo Nordisk). To knock down Itch in Hepa 1 cells, siRNAs against Itch (catalog 4390771, ID: S68413 and S68414) and control siRNA (catalog 4390843) were purchased from Thermo Fisher Scientific. Lipofectamine RNAiMAX Transfection Reagent (catalog 13778030) was used for siRNA transfection. Cell death was evaluated using the LDH Cytotoxicity Colorimetric Assay Kit II (BioVision, K313-500). LDH values were normalized to maximum releasable LDH activity after cell lysis.

Gene expression analysis. Total RNA from adipose tissues was extracted using a commercial kit from Invitrogen. RNAs from other tissues was extracted using the TRIzol method. For qPCR analysis, an equal amount of RNA was reverse-transcribed using MMLV-RT followed by qPCR reactions using SYBR green (Life Technologies). Relative abundance of mRNA was normalized to ribosomal protein 36B4. The primers for qPCR are listed in Supplemental Table 1.

For microarray study of liver gene expression, total liver RNA isolated from NASH diet-fed WT and Nrg4-KO mice was used to gen- erate probes for Affymetrix Mouse Gene ST 2.1 array strips. Sample processing and data analyses were performed according to the manufacturer's instructions. All original microarray data were deposited in the NCBI's Gene Expression Omnibus database (GEO GSE99753).

Immunoblotting analysis. For total lysates, livers were homogenized in a lysis buffer containing $50 \mathrm{mM}$ Tris ( $\mathrm{pH} 7.5), 150 \mathrm{mM} \mathrm{NaCl}$, $5 \mathrm{mM} \mathrm{NaF}, 25 \mathrm{mM} \beta$-glycerol phosphate, $1 \mathrm{mM}$ sodium orthovanadate, $10 \%$ glycerol, $1 \%$ Triton X-100, $1 \mathrm{mM}$ dithiothreitol (DTT), and freshly added protease inhibitors (Roche). Cells were harvested and homogenized with lysis buffer containing $2 \%$ sodium dodecyl sulfate (SDS), 50 mM Tris- $\mathrm{HCl}$ (pH 6.8), 10 mM DTT, 10\% glycerol, $0.002 \%$ bromphenol blue, and freshly added protease inhibitors. For protein stability assays, cells were treated with CHX (Sigma-Aldrich) before harvest. Immunoblotting experiments were performed using specific antibodies. For immunoblots, antibodies against c-FLIP ${ }_{\mathrm{L} / \mathrm{R}}$ (sc-5276) and HA-tag (sc-805) were from Santa Cruz Biotechnology Inc. Antibodies against CASP3 (catalog 9661), PARP (catalog 9542), phospho-ErbB4 (Y1284) (catalog 4757), total ErbB4 (catalog 4795), phospho-STAT5 (Y694) (catalog 9359), total STAT5 (catalog 9363), phospho-Akt (S473) (catalog 4060), total Akt (catalog 4691), phospho-JNK1/2 (T183/Y185) (catalog 4668), total JNK1/2 (catalog 9252), total Itch (catalog 12117), phospho-MLKL (S345) (catalog 62233), and phospho-MLKL (S358) (catalog 91689) were from Cell Signaling Technology. Antibodies against Flag-tag (F1904) and Tubulin (T6199) were from Sigma-Aldrich. 
Plasma and liver triacylglyceride analysis. The plasma ALT and AST levels were measured using kits from Stanbio, and plasma HMGB1 levels were measured using ELISA kits from antibodies-online (http:// www.antibodies-online.com/). Liver triacylglyceride (TAG) was extracted and measured as previously described.

Histopathologic analysis and immunofluorescence microscopy. Formalin-fixed, paraffin-embedded mouse liver sections were stained with $\mathrm{H} \& \mathrm{E}$ to evaluate steatosis and inflammatory cell infiltration. Liver fibrosis was assessed by Picrosirius (sirius) red (Polysciences, catalog 24901) staining of the formalin-fixed, paraffin-embedded mouse liver sections. TUNEL staining was conducted on the cryosections of the livers using a kit from BioVision (K401-60). For immunofluorescence microscopy, cryosections of the livers were fixed with $10 \%$ formalin, blocked with 5\% BSA (Sigma-Aldrich), and labeled with the antibody against mouse F4/80 (Abcam, ab16911) for 3 hours, followed by incubation with a fluorophore-conjugated secondary antibody (Thermo Fisher Scientific) for 1 hour. The stained sections were mounted with DAPI-containing mounting medium (VECTASHIELD) and then viewed on a Nikon A-1-A fluorescence microscope. For parameters involving sirius red staining and F4/80 immunofluorescence, computerized image analysis (ImageJ, NIH) was used to quantify the area stained as a percentage of the total area of view. TUNEL ${ }^{+}$cells were quantified (Image J) in TUNEL staining liver section images as the percentage of TUNEL ${ }^{+}$cells per total DAPI-stained cells. For lipid droplet number and size quantification, ImageJ was used on the H\&E staining images. The same threshold settings were used for all analyses.

Measurement of collagen content of liver tissue. Collagen content in the livers was evaluated by measuring the hydroxyproline level in the livers using the Hydroxyproline Colorimetric Assay Kit (K555100) from BioVision. Briefly, liver tissue was homogenized in water and samples were hydrolyzed by incubation with $6 \mathrm{~N}$ hydrochloric acid at $120^{\circ} \mathrm{C}$ for 3 hours. Liver hydrolysates were oxidized using chloramine-T, followed by incubation with Ehrlich's perchloric acid reagent for color development. Absorbance was measured at 560 $\mathrm{nm}$, and hydroxyproline quantities were calculated by reference to standards processed in parallel.

Ubiquitination assay. Hepa 1 cells were transfected with the plasmids encoding HA-tagged c-FLIP ${ }_{\mathrm{L}}$ and Flag-tagged ubiquitin by using polyethylenimine (Polysciences, catalog 23966). Ubiquitination assays were performed following a previously described protocol (46). Briefly, cells were lysed in 1\% SDS buffer (Tris [pH 7.5], 0.5 mM EDTA, $1 \mathrm{mM}$ DTT) and boiled for 10 minutes. For immunoprecipitation, the lysates were diluted 10 -fold in Tris- $\mathrm{HCl}$ buffer. Ubiquitination was assayed using anti-Flag immunoblotting.

Flow cytometry analysis. For cell death analysis, primary hepatocytes and Hepa 1 cells were digested with $0.05 \%$ Trypsin-EDTA (Gibco,
Thermo Fisher Scientific), washed with PBS, and then costained with annexin V/PI (V13242, Invitrogen) followed by flow cytometry. CD $4^{+} \mathrm{T}$ cell subpopulations in the liver were analyzed as described (47). Briefly, mouse livers were ground and lymphoid fraction in livers was isolated by density gradient centrifugation in Lymphoprep (AXS-1114547, AxisShield) followed by in vitro stimulation with phorbol-miristate acetate (PMA) (100 ng/ml, Sigma-Aldrich) plus ionomycin (1 $\mu \mathrm{M}$, SigmaAldrich) plus brefeldin A ( $1 \mu \mathrm{g} / \mathrm{ml}$, Sigma-Aldrich) for 4 hours. Cells were first stained extracellularly with specific antibodies against mouse CD4, CD8, CD45, and CD90.2 (BD Bioscience), were then fixed and permeabilized with Fixation/Permeabilization Solution (eBioscience), and finally were stained intracellularly with antibodies against mouse IL-17, TNF- $\alpha$, ROR $\gamma$ t, and FOXP3 (BD Biosciences). Samples were acquired on a flow cytometry analyzer (LSR II; BD Biosciences), and data were analyzed with DIVA software (BD Biosciences).

Statistics. All results are presented as mean \pm SEM. Student's $t$ test was used to analyze the differences between 2 groups, while 1-way ANOVA with post hoc Tukey's test was used to evaluate differences among groups when 3 or more groups were analyzed. A $P$ value of less than 0.05 was considered statistically significant. Statistical methods and corresponding $P$ values for data shown in each panel are included in the figure legends. All representative experiments were repeated at least 3 times.

Study approval. All animal studies were approved by the Institutional Animal Care and Research Advisory Committee at the University of Michigan. The use of human liver samples was approved by the Institutional Review Board at Northeast Ohio Medical University (NEOMED).

\section{Author contributions}

JDL and LG conceived the project and designed research. LG, PZ, ZC, HX, and SL performed the experiments. SK provided NRG4 recombinant protein for in vitro studies. LG, HX, JDL, YZ, and WZ analyzed the data. LG and JDL wrote the manuscript.

\section{Acknowledgments}

We thank the NASH Clinical Research Network for sharing human liver tissue samples and the staff at the University of Michigan sequencing core for performing microarray analysis. This work was supported by the NIH (DK102456 and AG055379 to JDL and CA211016 to WZ), Novo Nordisk through a research agreement, the Michigan Diabetes Research Center (DK020572), and the Michigan Nutrition and Obesity Center (DK089503).

Address correspondence to: Jiandie Lin, 5437 Life Sciences Institute, University of Michigan, 210 Washtenaw Avenue, Ann Arbor, Michigan 48109, USA. Phone: 734.615.3512; Email: jdlin@umich.edu.
1. Cohen JC, Horton JD, Hobbs HH. Human fatty liver disease: old questions and new insights. Science. 2011;332(6037):1519-1523.

2. Machado MV, Diehl AM. Pathogenesis of nonalcoholic Steatohepatitis. Gastroenterology. 2016;150(8):1769-1777.

3. Rinella ME. Nonalcoholic fatty liver disease: a systematic review. JAMA. 2015;313(22):2263-2273.

4. Hernandez-Gea V, Friedman SL. Pathogenesis of liver fibrosis. Annu Rev Pathol. 2011;6:425-456.

5. Marengo A, Rosso C, Bugianesi E. Liver cancer: connections with obesity, fatty liver, and cirrhosis. Annu Rev Med. 2016;67:103-117.

6. Michelotti GA, Machado MV, Diehl AM. NAFLD, NASH and liver cancer. Nat Rev Gastroenterol Hepatol. 2013;10(11):656-665

7. Heymann F, Tacke F. Immunology in the liver from homeostasis to disease. Nat Rev Gastroenterol Hepatol. 2016;13(2):88-110.

8. Neuschwander-Tetri BA. Hepatic lipotoxicity and the pathogenesis of nonalcoholic steatohepatitis: the central role of nontriglyceride fatty acid metabolites. Hepatology. 2010;52(2):774-788.

9. Sunny NE, Bril F, Cusi K. Mitochondrial adaptation in nonalcoholic fatty liver disease: nove mechanisms and treatment strategies. Trends Endocrinol Metab. 2017;28(4):250-260.

10. Tilg H, Moschen AR. Evolution of inflammation in nonalcoholic fatty liver disease: the multiple parallel hits hypothesis. Hepatology. 2010;52(5):1836-1846.

11. Brenner C, Galluzzi L, Kepp O, Kroemer G. Decoding cell death signals in liver inflamma- 
tion. J Hepatol. 2013;59(3):583-594.

12. Ding WX, Yin XM. Dissection of the multiple mechanisms of TNF-alpha-induced apoptosis in liver injury. JCell Mol Med. 2004;8(4):445-454.

13. Luedde T, Kaplowitz N, Schwabe RF. Cell death and cell death responses in liver disease: mechanisms and clinical relevance. Gastroenterology. 2014;147(4):765-783.e4.

14. Hatting M, et al. Hepatocyte caspase- 8 is an essential modulator of steatohepatitis in rodents. Hepatology. 2013;57(6):2189-2201.

15. Witek RP, et al. Pan-caspase inhibitor VX-166 reduces fibrosis in an animal model of nonalcoholic steatohepatitis. Hepatology. 2009;50(5):1421-1430.

16. Barreyro FJ, et al. The pan-caspase inhibitor Emricasan (IDN-6556) decreases liver injury and fibrosis in a murine model of non-alcoholic steatohepatitis. Liver Int. 2015;35(3):953-966.

17. Linkermann A, Green DR. Necroptosis. N Engl J Med. 2014;370(5):455-465.

18. Yuan J, Najafov A, Py BF. Roles of caspases in necrotic cell death. Cell. 2016;167(7):1693-1704.

19. Davidovich P, Kearney CJ, Martin SJ. Inflammatory outcomes of apoptosis, necrosis and necroptosis. Biol Chem. 2014;395(10):1163-1171.

20. Gautheron J, et al. A positive feedback loop between RIP3 and JNK controls nonalcoholic steatohepatitis. EMBO Mol Med. 2014;6(8):1062-1074.

21. Afonso MB, et al. Necroptosis is a key pathogenic event in human and experimental murine models of non-alcoholic steatohepatitis. Clin Sci. 2015;129(8):721-739.

22. Wree A, Mehal WZ, Feldstein AE. Targeting cell death and sterile inflammation loop for the treatment of nonalcoholic steatohepatitis. Semin Liver Dis. 2016;36(1):27-36.

23. Rosell M, et al. Brown and white adipose tissues: intrinsic differences in gene expression and response to cold exposure in mice. Am J Physiol Endocrinol Metab. 2014;306(8):E945-E964.

24. Wang GX, Zhao XY, Lin JD. The brown fat secretome: metabolic functions beyond thermogenesis. Trends Endocrinol Metab. 2015;26(5):231-237. 25. Wang GX, et al. The brown fat-enriched secreted factor Nrg4 preserves metabolic homeostasis through attenuation of hepatic lipogenesis. Nat Med. 2014;20(12):1436-1443.

26. Cai C, Lin M, Xu Y, Li X, Yang S, Zhang H. Association of circulating neuregulin 4 with metabolic syndrome in obese adults: a cross-sectional study. BMC Med.2016;14(1):165.

27. Dai YN, et al. A case-control study: association between serum neuregulin 4 level and nonalcoholic fatty liver disease. Metab Clin Exp. 2015;64(12):1667-1673.

28. Kim J, Lee T, Kim TH, Lee KT, Kim H. An integrated approach of comparative genomics and heritability analysis of pig and human on obesity trait: evidence for candidate genes on human chromosome 2. BMC Genomics. 2012;13:711.

29. Salinas YD, Wang L, DeWan AT. Multiethnic genome-wide association study identifies ethnic-specific associations with body mass index in Hispanics and African Americans. BMC Genet. 2016;17(1):78

30. Sandholm N, et al. New susceptibility loci associated with kidney disease in type 1 diabetes. PLoS Genet. 2012;8(9):e1002921.

31. Oztürk S, Schleich K, Lavrik IN. Cellular FLICElike inhibitory proteins (c-FLIPs): fine-tuners of life and death decisions. Exp Cell Res. 2012;318(11):1324-1331.

32. Tsuchiya Y, Nakabayashi O, Nakano H. FLIP the Switch: Regulation of Apoptosis and Necroptosis by cFLIP. Int J Mol Sci. 2015;16(12):30321-30341.

33. Xu Y, Zalzala M, Xu J, Li Y, Yin L, Zhang Y. A metabolic stress-inducible miR-34a-HNF4 $\alpha$ pathway regulates lipid and lipoprotein metabolism. Nat Commun. 2015;6:7466.

34. Clapper JR, et al. Diet-induced mouse model of fatty liver disease and nonalcoholic steatohepatitis reflecting clinical disease progression and methods of assessment. Am J Physiol Gastrointest Liver Physiol. 2013;305(7):G483-G495.

35. Giles DA, Moreno-Fernandez ME, Divanovic S. IL-17 Axis driven inflammation in non-alcoholic fatty liver disease progression. Curr Drug Targets. 2015;16(12):1315-1323.

36. Carver RS, Stevenson MC, Scheving LA, Russell WE. Diverse expression of ErbB receptor pro- teins during rat liver development and regeneration. Gastroenterology. 2002;123(6):2017-2027.

37. Ni HM, McGill MR, Chao X, Woolbright BL, Jaeschke H, Ding WX. Caspase inhibition prevents tumor necrosis factor- $\alpha$-induced apoptosis and promotes necrotic cell death in mouse hepatocytes in vivo and in vitro. Am J Pathol. 2016;186(10):2623-2636 .

38. Yang L, et al. Transforming growth factor beta signaling in hepatocytes participates in steatohepatitis through regulation of cell death and lipid metabolism in mice. Hepatology. 2014;59(2):483-495.

39. Piao X, et al. c-FLIP maintains tissue homeostasis by preventing apoptosis and programmed necrosis. Sci Signal. 2012;5(255):ra93.

40. Safa AR. Roles of c-FLIP in apoptosis, necroptosis, and autophagy. JCarcinog Mutagen. 2013;(Suppl 6):003.

41. Wang PX, et al. Targeting CASP8 and FADD-like apoptosis regulator ameliorates nonalcoholic steatohepatitis in mice and nonhuman primates. Nat Med. 2017;23(4):439-449.

42. Chang L, et al. The E3 ubiquitin ligase itch couples JNK activation to TNFalpha-induced cell death by inducing c-FLIP(L) turnover. Cell. 2006;124(3):601-613.

43. Malhi H, Guicciardi ME, Gores GJ. Hepatocyte death: a clear and present danger. Physiol Rev. 2010;90(3):1165-1194.

44. Geserick P, et al. Cellular IAPs inhibit a cryptic CD95-induced cell death by limiting RIP1 kinase recruitment. JCell Biol. 2009;187(7):1037-1054.

45. Feoktistova M, et al. cIAPs block Ripoptosome formation, a RIP1/caspase- 8 containing intracellular cell death complex differentially regulated by cFLIP isoforms. Mol Cell. 2011;43(3):449-463.

46. Guo L, et al. Acetylation of mitochondrial trifunctional protein $\alpha$-subunit enhances its stability to promote fatty acid oxidation and is decreased in nonalcoholic fatty liver disease. Mol Cell Biol. 2016;36(20):2553-2567.

47. Zhao E, et al. Cancer mediates effector $\mathrm{T}$ cell dysfunction by targeting microRNAs and EZH2 via glycolysis restriction. Nat Immunol. 2016;17(1):95-103. 